\title{
STUDY OF EQUILIBRIUM AIR TOTAL RADIATION ${ }^{1}$
}

by
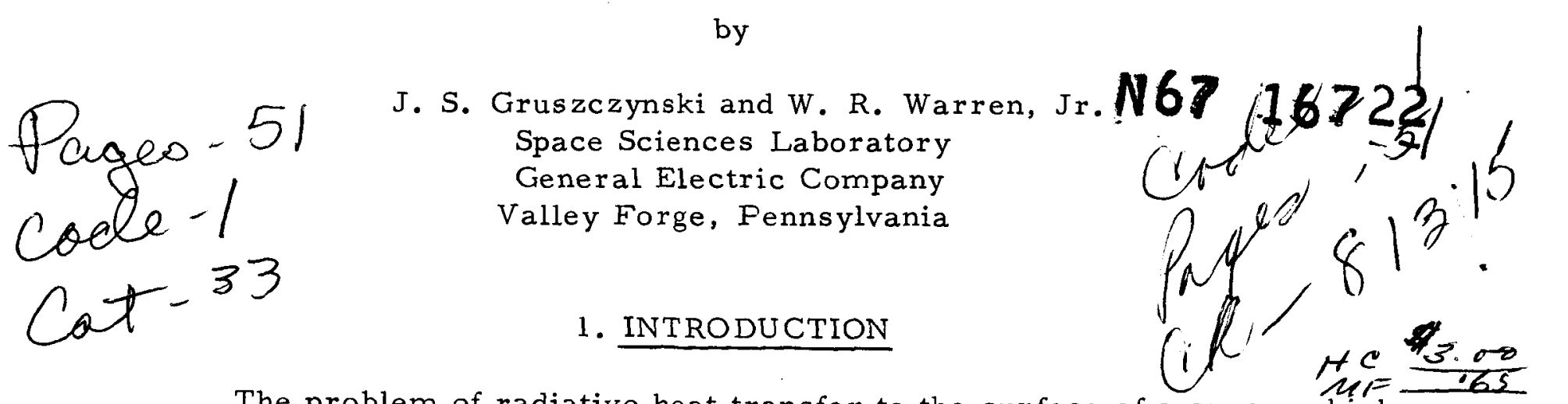

The problem of radiative heat transfer to the surface of a space vehicle

during entry into a planetary atmosphere has gained in importance with the establishment of national programs for lunar exploration and missions to the near planets. The entry velocities for some of these missions will be well above the Earth orbital value and, therefore, the flow in the stagnation region of a blunt vehicle will reach high pressure and temperature levels. For example equilibrium stagnation temperatures of more than $14,000 \mathrm{~K}$ will be reached for the re-entry from a Mars mission. Such gas can be expected to emit large amounts of radiant energy characteristic of the chemical species present in the flow.

In considering the contribution of radiative transport processes to the heat transfer experienced by an entry vehicle, a distinction can be made between equilibrium radiation originating in the shock-processed gas, which after passing through the bow wave has relaxed to its thermochemical equilibrium state, and the non-equilibrium radiation, which is emitted from the shock front in which the imparted energy has not been distributed among the various degrees of freedom. This paper is concerned only with equilibrium radiation in air and gas mixtures assumed to represent the atmospheres of the near planets.

1. Supported by the NASA Office of Advanced Research and Technology under Contract NASw-939. 


\section{THEORETICAL CONSIDERATIONS}

The radiance of high temperature air is a result of several complex physical processes and, although the fundamentals have been studied theoretically in detail, its prediction depends to a considerable degree on approximating assumptions. Therefore, experimental data are required to evaluate the predictions. The primary objective of this paper is to present the results of a shock tube experimental study designed to obtain total air radiance data at temperature and pressures of interest to hypervelocity entry. The unique feature of this work is the development and use of a fast response total radiation cavity gage system that permits the sensing of energy radiated from a model shock layer through a non-absorbing optical path (measurements with controlled absorption are also possible). Thus, the important contribution of the vacuum UV region of the spectrum to the total radiant flux is not blocked by the measurement system. Before describing the experimental study, however, we will discuss some of the theoretical aspects of high temperature air radiation.

Because of the early interest in orbital and sub-orbital re-entry, considerable attention has been given in the past to the prediction of the radiance of air and similar gases at relatively long wavelengths - greater than about $1600 \AA$ - and several experimental studies have been made to evaluate these results. At the high temperatures (above about $8000^{\circ} \mathrm{K}$ ) and density levels of interest in superorbital entry, however, radiation at shorter 
wavelengths tend to become important, both because of the shift of the blackbody curve in this direction and the relatively high absorption coefficients of atomic processes active there. This was indicated in the theoretical results of Breene and his associates $(4)^{1}$; the implications of Breene's predictions in terms of radiative heat transfer at superorbital flight conditions were discussed by the present authors in Ref. 5 .

In Breene's analysis, the major contributor to air radiance for $\lambda<1600 \stackrel{\circ}{A}$ is the electron-atomic ion recombination (free-bound) mechanism. Breene's simplified analytical model for this mechanism has been improved upon in the recent works of Sherman and Kulander (6) and Hahne (7). In Fig. 1 we have shown the $\lambda .1600 \mathrm{~A}$ intensity predictions of Breene and of Sherman and Kulander, each added to the Breene $\lambda$ ' $1600 \AA$ calculations so that the importance of the vacuum UV contribution can be seen in terms of a nominal total intensity. The results in Fig. 1 are for a blunt model shock tube flow over a range of shock velocities and for a single initial tube pressure and a shock stand-off value; the relationship between shock and flight velocity for this flow configuration is shown on the abcissa. It is seen that at the se fairly high density conditions (corresponding to a simulated flight altitude of approximately $130,000 \mathrm{ft}$ ) the $\lambda<1600 \AA$ A contributions are predicted to account for about half of the total intensity. For low density levels or a thinner layer the relative contributions of the vacuum UV will increase because of the self-absorbing properties of the gas.

1. Numbers in parentheses refer to references listed at end of paper. 
Biberman and his associates have also conducted detailed studies of radiative heat transfer from air including mechanisms in the vacuum $U V$. A recent summary of their work is given in Ref. 8. Unfortunately, these results are presented quantitatively only in terms of integrated radiative heat transfer values so that the contributions of the different mechanisms to the total gas radiance can only be approximately deduced. Perhaps the most significant result of this work is the indication that atomic line radiation - mostly in the vacuum UV - will account for more than $50 \%$ of the total gas radiance over wide ranges of flow properties and radiating layer thicknesses of interest in re-entry flight.

An important characteristic of the vacuum UV radiating mechanisms considered by the above investigators is their high absorption coefficient level. This causes the predicted vacuum UV radiation to be strongly selfabsorbed within a shock layer at flight conditions of interest while the same layer will be essentially transparent to radiation at longer wavelengths. Thus, at a given velocity and altitude a thin layer might be dominated by the vacuum UV contribution and a thick layer by the long wavelength radiation. The predicted effects of self-absorption on radiative heat transfer are shown in Figs. 2 and 3. In Fig. 2 we have presented the total equilibrium radiative heating predictions of Ref. 8 for a range of $R_{N}$ values and over a range of flight velocities and altitudes. The predictions of Serbin (9) were used to determine the shock detachment distance and no correction was made to 
account for the curvature of the shock layer. The theoretical data have been normalized by $R_{N} \cdot 6$ and it is seen that this is a reasonably good correlation parameter. For a shock layer transparent to all wavelengths, the correlation parameter would be simply $R_{N} \cdot$ Thus, the overall effect on total radiative heating of self-absorption is reasonably well approximated by assuming that the radiation is proportional to $\mathrm{R}_{\mathrm{N}}^{\cdot 6}$ (for this flight regime and the assumptions of the theoretical results, of course). Note that this is simply a correlation property of detailed analytical results and does not justify assumptions of the type that assign a wavelength averaged property to the gas (such as Planck mean absorption coefficient or emissivity) and uses this property for further analysis; that is, the fact that the absorption coefficient is a strong function of wavelength must, in general, be factored into any detailed analysis of radiative transfer through, or out of, a high temperature gas region.

Fig. 3 allows us to compare the predictions of different authors and assess the importance of absorption on the radiative heat transfer for a hypothetical flight situation. The upper two values were calculated from the Breene absorption coefficients for the entire wavelength range of interest. The effect of absorption was included in the second; it reduces appreciably the vacuum UV heating but has a negligible effect on the long wavelength contribution. As discussed earlier (Fig. 1), the Sherman and Kulander vacuum UV prediction is lower than Breene's. The Biberman prediction, taken from Fig. 2, includes the self-absorption effect. It is somewhat more 
than twice the Breene-Sherman-Kulander value which does not include line contributions; therefore, considering the importance of line radiation in the Biberman results, we can conclude that, at least for this flight situation, there is an approximate agreement between the Biberman, et al and the Breene-Sherman-Kulander predictions for those mechanisms considered in the latter analysis.

In addition to self-absorption, we must consider the fact that the thermal radiation from the shock layer will reduce the total energy level of the gas and hence its temperature thus causing the emitted energy to be lower than that calculated on an isoenergetic shock layer assumption. This can be referred to as the coupling of radiation and convection (10). Results of analytical studies of this effect have been presented by Goulard (10), Howe and Viegas (11), Hoshizaki and Wils on (12), Wilson and Hoshizaki (13) and the results of Refs. 11 and 13 have been compared in Ref. 12. These theories assumed that the gas was either transparent (10) (12) (13) or a grey body (11) (they did not treat the self-absorption characteristics as a function of wavelength), so that their results must be considered to be only an approximation of the magnitude of coupling effects. Recently Hoshizaki and Wilson (14) and Sherman (15) developed theoretical approaches to the radiating shock layer problem including the effects of coupling with wavelength dependent absorption and including the effects of viscosity.

It is anticipated that, when the energy radiated away from the shock layer becomes a large fraction of the energy convected by the flow, important 
coupling effects will be present. A parameter defined as the ratio of the uncoupled energy radiated from a heated gas region to the flow energy flux through that region is often used to indicate such conditions. For a thin shock layer, this ratio can be defined as

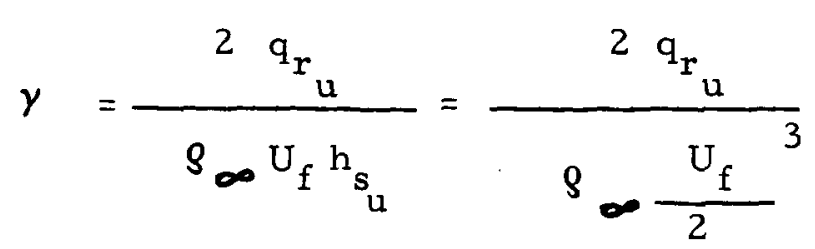

We have indicated lines of constant on Fig. 2 for two spherical body nose radii. It is seen that for bodies with $R_{N}>1 \mathrm{ft}$, the value of $\gamma$ can be of the order of 1 at velocities below the escape value. Since this value of $\gamma$ represents a large decrease (25\% or more) in radiative heating according to the theories mentioned above, we can be concerned that coupling effects might be significant in important flight situations.

Intuitively, it seems reasonable to expect that the reduction in radiative heat transfer due to coupling for a blunt body flow will be primarily a function of $\gamma$, at least for the transparent gas assumption. To check this we have conducted a simple analysis for shock layer thickness range of 1 to $10 \mathrm{~cm}$. First, the Biberman $q_{r_{u}}$ values were calculated for a given flight condition; second, the radiated energy loss was assigned uniformly across the shock layer (isothermal assumption) resulting in a lower average stagnation region enthalpy; third, a $\mathrm{q}_{\mathbf{r}_{c}}{ }^{\prime}$ value for the new effective stagnation conditions was calculated; and fourth, the procedure was iterated until $q_{\mathbf{r}_{c}}$ 
was determined with reasonable accuracy. Following this approximate procedure, it is indeed found that the percentage radiative heating reduction apparently is only a function of $\gamma$. This result is given in Fig. 4. Also shown in the same figure are several results from Refs. 11 and 12 (as given in the latter). The good agreement between these results and the present analysis fortifies the conclusion that $\gamma$ is the important variable and suggests that the assumptions used in the three calculation procedures are equivalent. Different results are presented in Ref. 10 which predict considerably more reduction in radiative heating as a function of $\gamma$ than shown in Fig. 4 (e.g., $\mathrm{q}_{\mathrm{r}_{\mathrm{c}}} / \mathrm{q}_{\mathrm{r}_{\mathrm{u}}}=.5$ at $\left.\gamma=.05\right)$. Finally, we again emphasize that these conclusions are not necessarily valid for a gas that has a strong wavelength dependence of its optical properties which will tend to produce temperature variations across the shock layer.

An interesting property of the coupling parameter can be seen through analysis of a hypothetical entry vehicle trajectory. Fig. 5 shows the velocityaltitude trajectory (simple ballistic) of a $R_{N}=1 \mathrm{ft}$. body that enters the atmosphere at 40,000 ft/sec. We have also shown the uncoupled heating predictions of Biberman and the coupling parameter based on the se predictions. From the shape of these curves, it can be seen that location of the peak coupling parameter precedes the peak uncoupled radiative heating point by several seconds. This is seen in Fig. 6 where $\gamma$ and $\mathrm{q}_{\mathrm{r}}$ are plotted as functions of time. If we now make the assumption, discussed above, that the percentage reduction in radiative heating at any point on the trajectory is 
only a function of $\gamma$, we conclude that the coupling effect will not only reduce the radiative heating but also shift the maximum heating point (and the centroid of the heating pulse area) somewhat to later times than indicated by an uncoupled prediction. This property is illustrated in Fig. 6 through plots of the coupled heating, $\mathrm{q}_{\mathrm{r}}$, as determined from Fig. 4 and Ref. 10 again assuming that $\mathrm{q}_{\mathrm{r}_{\mathrm{c}}} / \mathrm{q}_{\mathrm{r}_{\mathrm{u}}}$ is only a function of $\gamma$. The approximate coupling correction to the Biberman prediction, based on Ref. 10 , is also shown in Fig. 3.

From this brief discussion we conclude that radiative contributions in the vacuum UV region of the spectrum (nominally defined here as 500 to 1600 A ), self-absorption of the shock layer gas, and coupling between the radiated energy and the flow energy are of potential importance in the study of radiative heating to entry vehicles in superorbital flight regimes. In a sense, the existence of strong vacuum UV radiation promotes the importance of self-absorption and coupling, since it is the radiation at these wavelengths that has large absorption coefficients, and since the coupling parameter at a given velocity-altitude point is proportional to the total energy radiated away. On the other hand, the coupling problem is relatively much more amenable to analysis if the gas is transparent.

The following sections present a description and the results of our experimental study. In this paper we have concentrated on determining the importance of the vacuum UV contribution; however, we have also investigated the ability of the shock tube to provide useful flow conditions well into the 
coupled regime and obtained some preliminary indication of the importance of coupling at a $\gamma$ value of the order of . 1 .

\section{EXPERIMENTAL TECHNIQUES}

Facility Description and Performance

In the study of radiative properties of high temperature gases one is confronted with the problem of producing well defined samples of test gas at appropriate temperature and pressure levels for time durations compatible with the response characteristics of the radiation sensors. In the case of model measurements, this must be long enough to allow the formation of a steady flow around the model. The simulation of conditions corresponding to hypervelocity entry has become possible with the development of the electrically driven shock tube (16) (17). It is essentially a conventional shock tube in which the driver gas remains isolated from the test gas in the low pressure driven section by means of a metal diaphragm until the heating process of the light gas in the driver - an electrical discharge of capacitor stored energy - is completed. The design features and the mode of operation have been discussed in Refs. 5 and 18. The driven section has a 6 in. internal diameter and is $31.5 \mathrm{ft}$. long. A capacitor bank rated at 304,000 joules supplies the energy to the driver which may be varied in size up to a 3 in. diameter, and $41 / 2 \mathrm{ft}$. length.

Fig. 7 illustrates the instrumentation techniques used to determine the shock tube performance; also indicated are the techniques used to measure 
total gas radiance both behind the incident shock wave and in the stagnation region of a blunt model in the flow between the incident shock wave and the driver gas. This paper will present results only from the studies in the blunt model flow.

To determine the test flow properties and to assure validity of the data during each run, several measurements are made. Signals from a series of photomultipliers located at various stations along the shock tube are used to measure the incident shock wave velocity as a function of distance from the diaphragm. From this and the initial shock tube pressure, and with the help of the thermochemical equilibrium shock tube relations the properties in a particular flow region are determined. The techniques used for assessing the quality of the test flow are dependent on observations (from the sidewall of the shock tube) of the radiant emission from the gas behind the incident shock wave and from the flow in the stagnation region of the model. Since the emission is a strong function of temperature, the temporal quality of the flow, in terms of its thermodynamic properties, and its duration can be deduced from the steadiness of the emitted radiation, while the spatial quality, from the image converter photo raphs. Instrumentation details and shock tube performance data are discussed in Refs. 3, 5, 16,18 and 19.

In view of the discussions in the last section, it is interesting to consider shock tube performance in terms of flight condition simulation and coupling parameter levels. This has been done in Fig. 8. Shown are sets of 
incident shock velocity - initial tube pressure data taken with different size driver tubes (represented by the nominal driver energy density values). Scales showing flight velocity and approximate altitude simulation achieved in the blunt model stagnation region are given on the figure. Also shown are plots of the coupling parameter, $($, based on the Biberman, et al predictions, for a flat faced cylindrical model with a 1.5 in. diameter. A uniform test time of about $9 \mu$ s was obtained for the high velocity runs in Fig. 8. More than half of this can be considered to be the quasi-steady test time during which test data can be obtained. The time responses of the measurement techniques used are well within such a flow duration. For lower velocity runs, the test durations are usually considerably longer than that shown in Fig. 8 (see Refs. 5, 18 and 19). Therefore, it can be concluded that the test facility used for the present study is capable of producing useful test flows to simulated flight velocity levels of $60,000 \mathrm{ft} / \mathrm{sec}$. At these conditions and for model sizes that have been used, values of $\gamma$ considerably greater than .1 are obtained. This permits further study of the important effects of radiative-convective energy coupling on the stagnation region flow. Also, since there is adequate test time available at the $U_{f}=$ $60,000 \mathrm{ft} / \mathrm{sec}$ condition, it seems reasonable to assume that significantly higher flight velocity simulation with useful test durations would be available in the present facility through the use of a higher energy density driver. 
Total Radiation Measurement Techniques

The method followed here for the experimental study of high temperature gas radiation was based upon the development of a measuring technique capable of sensing integrated total radiance over the complete wavelength spectrum of interest. Photo-emissive devices in general use, such as photomultipliers and phototubes, are sensitive only over a limited range of wavelength. A thin film resistance thermometer gage of the type widely employed in shock tubes and shock tunnels for measuring convective heat transfer offers suitable sensitivity and fast response. However, a difficulty arises in the determination of its surface reflectivity which is a function of the surface conditions, the wavelength of the incident radiation and the incident angle.

The gage used in the present experiments (20) draws on the blackbody cavity principle for its geometric shape. The gage is made in the form of a cylindrical body with the entrance slit set off-axis. The interior of the gage is coated with a thin film of platinum which serves as a fast response resistance thermometer. Because of the chosen geometry almost all radiation which enters the gage is trapped inside giving it a response which is essentially independent of the radiation wavelength.

The temperature and density level of the gas under study can cause a wide variation in the spectral distribution of the emitted radiation. In Fig. 9 theoretical spectral distributions of air radiance are shown, one for air at $7000^{\circ} \mathrm{K}$ and 1 atm (4) and the other at $14,000^{\circ} \mathrm{K}$ and the same pressure 
but without the contribution from line radiation (8). The spectrum at the lower temperature contains mostly contributions from molecular bands in the UV, visible and IR range. Very little emission is expected below $1800 \AA$ due to the limitations of the black body radiation. At the higher temperature, however, free-bound, free-free and bound-bound transitions in the vacuum UV part of the spectrum are responsible for a larger part of the total emission.

Materials normally used for windows in shock tubes have distinct short wavelength transmission cut-offs. These cut-off values, as shown in Fig. 9, occur at wavelengths longer than much of the radiation of interest in high temperature gas radiance studies. Therefore, a measurement system with a windowless capability is suggested. The remainder of this Section is concerned with the steps leading to the development of cavity gage-model systems with and without solid windows in the gage-togas optical path. Data presented in this paper were measured using the stagnation region gas ahead of a blunt model as the test gas sample. The arrangement of the total radiation cavity gage inside a model with a window was described in Ref. 5. Several different window materials - quartz, sapphire, lithium fluoride - are being used in this measurement system. Therefore, the combination of the gage-model system and the short $\lambda$ cut-off wavelengths of the window materials (including the gases used in the windowless configuration discussed below) offer a reasonable degree of spectral selectivity for this measuring system (in addition to its total capability). 
During initial operation of the gage, evidence of photoemission from the platinum film was observed. For tests in which the gage was evacuated to a pressure of approximately 3 microns, the photoelectric effect was strong enough to produce partial short-circuiting of the gage. A few runs were made with a glass window under the same flow conditions with no evidence of the photoelectric effect; this indicated that the photons with energy corresponding to a wavelength smaller than about $3500 \AA$ A are required to cause the photoemission (as expected). To further investigate this effect, the gage was filled with pure nitrogen at 1 atm pressure. It was hypothesized that the nitrogen would reduce the mean free path of the electrons and cause a space charge to develop close to the surface of the gage, which would inhibit any further electron emission within a fraction of a microsecond. Nitrogen gas was chosen initially because of its large cross-section for collision with electrons.

The use of $\mathrm{N}_{2}$ is satisfactory behind sapphire and quartz windows; however, for studies of vacuum UV radiation, $\mathrm{N}_{2}$ is not suitable because of its photoabsorption characteristics (see Fig. 9). In this case, the choice is limited to rare gases, with helium and argon displaying the most desirable optical properties. (The cut-off for helium, not shown in Fig. 9, occurs at about $190,000 \mathrm{~cm}^{-1}$ ). Although argon and krypton display cross-sections for electrons greater than nitrogen at electron energies above $4 \mathrm{ev}$, both are practically transparent to electrons with energies in the vicinity of $1 \mathrm{ev}$. This transparency, known as the Ramsauer-Townsend effect, is typical of 
the heavier rare gases. A gas mixture with equal proportions of $\mathrm{Kr}$, having large cross-sections for energetic electrons, and $\mathrm{He}$, with a reasonable effectiveness for scattering slow electrons, was found to supress the photoelectric effects.

As previously mentioned, a windowless gage-model system is required for sensing vacuum UV radiation. Since a gas mixture within the model is necessary to counteract the photoelectric effect, the problem arises of how to contain this gas inside the model prior to the arrival of the incident shock wave and prevent it from mixing appreciably with the test gas. The results obtained earlier indicated that the gas density inside the model must be considerably higher than the shock tube initial pressure for an effective restriction of the movement of the photoelectrons. A windowless model as shown in Fig. 10 was therefore developed in which a stretched latex membrane separates the model gas from the shock tube. A pulse of current is passed through a .002 in. wire located along the rectangular entrance slit and in contact with the latex membrane. No combustion of the membrane takes place but the rapid heating weakens the latex to a point where it tears under its own internal stress. The process of the uncovering of the entrance slit of the model takes approximately $15 \mu \mathrm{s}$. Depending on the gage gas pressure, a delay of about $140 \mu$ s between the current pulse and full opening of the slit was observed. This delay was found to be repeatable within $\pm 10 \mu \mathrm{s}$. By using a delayed trigger signal from an upstream station, the full opening process can be scheduled to be completed approximately $10 \mu \mathrm{s}$ prior to the 
arrival of the incident shock wave at the model station. Consideration of the inflow process in the case when the internal pressure is much lower than the stagnation pressure, indicated that it would be difficult to account properly for the absorption characteristics of the inflowing gas (unsteady and non-equilibrium expansion process in the optical path). If the internal pressure is set equal to the stagnation pressure this effect will ideally be eliminated; therefore, we have followed the latter approach in our windowless system.

At the instant when the latex membrane breaks, the gage gas issues through the entrance slit into the stationary gas in the shock tube, Since the breaking point of the membrane occurs approximately $10 \mu \mathrm{s}$ before the incident shock arrives at the model, only a small amount of the gage gas escapes and its penetration is limited to a few centimeters upstream of the model. This gas is swept downstream during the first $1-2 \mu$ s after the incident shock arrives the model which is usually less than the time needed to form the quasi-steady blunt body flow. Another advantage of being able to time the membrane opening to within about $10 \mu \mathrm{s}$ of the shock arrival is that the expansion wave inside the model does not reach the gage and cause a convective perturbation within the flow test time. Assuming that the interface between the test gas and the gage gas is stationary, the diffusion of the gases across it was estimated to be negligible during the time corresponding to a typical test gas flow $(20-30 \mu \mathrm{s})$. An oscilloscope trace of the gage signal obtained from the windowless model is shown in Fig. 10. In this case the 
interior of the model was filled with a mixture of one to three ratio of $\mathrm{He}$ and $\mathrm{Kr}$.

Note that the use of $\mathrm{Kr}$ as a gas window does not really permit the sensing of total gas radiance, since the low wavelength cut-off of $\mathrm{Kr}$ (photoionization) is approximately $880 \stackrel{\circ}{\AA}$. Depending on the theoretical estimates used, the Kr may block $10-20 \%$ (Biberman, et al) to 25-30\% (Breene-Sherman and Kulander) of the total radiance in a shock tube test at $14000^{\circ} \mathrm{K}$. However, the spectral region between $880 \AA$ and $1200 \AA$ is of considerable importance according to the predictions discussed earlier. Therefore, we believe that the windowless gage-model system as presently developed represents a useful step towards the achievement of a true total gas radiance sensor. Photographs of the shock layer ahead of the blunt model are taken during each run with an image converter camera. These data are required to establish the volume of the radiating gas sample that contributes to the radiant energy sensed by the cavity gage. Photographs from a run are shown in Fig. 11, with the three consecutive frames taken $5 \mu \mathrm{s}$ apart. The lower traces in the same figure show the camera monitor and photomultiplier signals. The photomultiplier looks through a window in the sidewall of the shock tube and is focused just ahead of the model stagnation point. The first image converter camera frame was obtained during the flow formation as indicated by the camera monitor. The next two frames were taken during the steady flow corresponding to an approximately uniform shock layer 
radiance and constant stand-off distance. These photographs also indicate that a uniform flow was achieved within the expected time (with the end of test gas flow clearly visible), and that the shape of the bow shock wave was not perturbed by the presence of the windowless slit at the stagnation point of the model.

\section{RESULTS}

Data obtained from the shock tube blunt model test flow using the window and windowless cavity gage measurement techniques described in the last section are shown in Figs. 12 and 13. Compared in Fig. 12 are results for $11 / 2$ inch diameter flat faced model with quartz windows and with the window-less configuration (with an internal $\mathrm{He}-\mathrm{Kr}$ mixture) and results for a $R_{N}=1$ inch hemispherical model with quartz windows. In Fig. 13, we have compared data obtained with quartz and LiF windows. On both figures, we have shown the intensity predictions for the shock tube conditions of Breene, et al $(\lambda>1600 \AA$ A $)$, Breene $(\lambda>1600 \AA$ A $)$ Sherman and Kulander $(\lambda>1600 \AA$ A $)$, assuming a $1 \mathrm{~cm}$ stand-off distance. An estimate for line radiation has been made using Vorobev and Norman (21) predictions which has to be given as an approximate band since their results did not coincide with the conditions of our experiment. The data in Figs. 12 and 13 have also been referenced to a $1 \mathrm{~cm}$ thick shock layer value from the shock stand-off distance measurements of $2.8 \mathrm{~mm}$ for the hemispherical model test and $7.6 \mathrm{~mm}$ for the flat faced model. This allows the results to be more easily compared. A theoretical correction of $9 \%$ - due to self- 
absorption - has been applied to the windowless data to account for this adjustment. The indicated corrections to the window data are negligible. A theoretical correction of $10 \%$ has also been included in the windowless data to account for the low length radiation cut-off by $\mathrm{Kr}$ in the model. Finally, a conservative estimate of self-absorption in the stagnation region boundary layer was made at the velocity level of the windowless tests and at a potentially severe absorption density level. An apparent reduction in intensity of $8 \%$ was indicated for these conditions; however, no correction for this effect has been made in the data.

First, in Fig. 12 it is seen that the windowless data are considerably higher than those from the window models. The two corrections to the data are in opposite directions so that their relative positions in the figure are equivalent to the relative levels of radiant energy sensed by the gages in the shock tube tests. Therefore, the data show a large contribution to the radiant intensity between the low wavelength cut-offs of quartz and of $\mathrm{Kr}$. Second, the quartz data are in reasonably good agreement with the Breene's

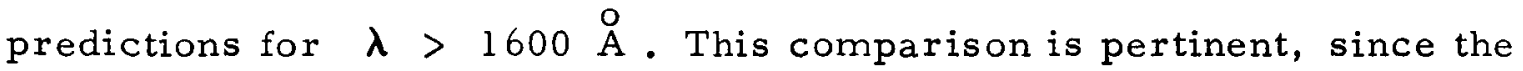
quartz windows had a relatively low cut-off wavelength (calibration of several samples of those used in our tests showed $80 \%$ transmission at $1700 \AA$ A) and since there is a negligible contribution to intensity in Breene's predictions between $1600 \AA$ and $1700 \AA$. Nerem (22) has compared experimental equilibrium intensity results for air of several investigators (1) (23) (24) taken with solid windows with cut-off levels to as low as $1700 \stackrel{\circ}{\mathrm{A}}$. He has found reasonably 
good agreement between these results and between his data and the high wavelength predictions of Breene. Good agreement with Breene's high wavelength predictions for a gas mixture of $91 \% \mathrm{~N}_{2}-9 \% \mathrm{CO}_{2}$ was also found in Ref. 5 over a similar test condition region. Third, the windowless data are in apparent good agreement with the Biberman, et al pre dictions based on the estimates derived from Ref. 21. Fourth, the agreement between the quartz data for the different shock stand-off distances indicates that there is a negligible self-absorption effect at long wavelengths (as expected, of course). More importantly, since the intensity data for the small stand-off distance should be larger than those for the larger value if coupling effects are present, these results imply that the effect of coupling is small for the present test conditions $(\gamma$ is the order of .1 for the flat faced body - see Fig. 11). Of course, an effect of the order predicted in Fig. 4 could be hidden within the scatter of the data in Fig. 12. Therefore, conclusions on the importance of coupling in a radiating flow based on the present data must be considered to be preliminary.

In Fig. 13, the quartz and lithium fluoride data comparison suggests a somewhat higher intensity level in the latter. However, we found LiF to be a difficult material to work with; the quality and, possibly, the cut-off wavelength can vary from sample to sample. Examination of the windows revealed that they became crazed after each run. The latter problem is of particular importance, since we have not been able to determine if the crazing 
occurs during the test time. Therefore, we believe that a definite conclusion cannot be drawn now from our data concerning the intensity in the region between the (potentially available) $1100 \stackrel{\circ}{\mathrm{A}}$ low wavelength cut-off usually quoted for LiF and that for quartz. Nerem has presented several data obtained with LiF windows and concluded that there is no substantial contribution to the total radiation due to emission in the wavelength region of 1200 to $1700 \stackrel{\circ}{\mathrm{A}}(22)$.

\section{CONCLUSIONS}

Results have been presented from a study of high temperature air radiance over range of equilibrium gas properties of interest to superorbital re-entry vehicles.: Several theoretical approaches to the problem have been reviewed and compared; the following points appear to be of interest: 1) radiation in the vacuum UV region of the spectrum (defined here to be $\lambda<$

$1600 \stackrel{\circ}{\mathrm{A}}$ ) can be expected to be important; 2) because of the large cross section of the radiative mechanisms in the vacuum UV, self-absorption in a radiating gas layer must be included in the analysis of energy fluxes through such a layer with a proper regard for the wavelength dependence of the absorption coefficient; 3) coupling between the radiated and flow energy fluxes may significantly reduce the total radiative heat transfer in comparison to that calculated for an isoenergetic layer; however, the prediction of such an effect is complicated by the wavelength dependent self-absorbing properties of the gas; 4) three different theoretical predictions (4) (6) (7) of electron-ion re- 
combination radiance accounts for a difference of up to $30 \%$ in total radiative heat transfer for the cases considered (not including line contributions);

5) there is an approximate agreement between the predictions of Biberman, et al and the other theoretical investigators considered here for mechanisms excluding line radiation, which, however, accounts for more than $50 \%$ of the Biberman, et al total prediction; 6) a body scale parameter, $R_{N} 6$, has been found to approximately correlate the Biberman total uncoupled radiative heat transfer predictions; the difference between this dependence and that for a simple transparent shock layer gas, ${ }_{N}$, indicates the integrated influence of self-absorption in the shock layer; 7) the predictions of Biberman result in relatively high values of the coupling parameter $(\gamma=.1)$ for body dimensions of interest at velocities of the order at the Earth escape value; and 8) for a simple type of entry vehicle, $\gamma$ will maximize before the peak uncoupled heating point with the probable result not only in a reduction of radiative heating but also in a shift of the maximum radiative heating point to later times in the trajectory.

A shock tube experimental study has been conducted to investigate several of the total radiance properties of air. It has been shown that an electrically driven "conventional" shock tube is capable of producing useful model stagnation region test flows to the $60,000 \mathrm{ft} / \mathrm{sec}$ flight velocity simulation level. At such conditions the $\gamma$ values for models of practical size are far in excess of .1 , and the refore, important combined effects of self- 
absorption and coupling are to be expected. A fast response total radiance measurement technique that employs a thin film cavity sensing element has been developed and used in the study. The cavity gage has been combined with blunt shock tube models that permit the sensing of energy radiated from the shock layer with and without solid windows in the optical path. In its windowless configuration, the measurement system as presently developed has a short $\lambda$ cut-off of approximately $880 \AA$. This represents a considerable improvement as compared to the best available solid windows and, according to the available predictions, should permit the sensing of most of the radiated energy at the conditions of interest.

Experimental gas radiance data, obtained in the vicinity of a simulated $38,000 \mathrm{ft} / \mathrm{sec}$ flight velocity, show that there is a significant contribution to intensity in the vacuum UV portion of the spectrum. The windowless data tend to agree with the available total radiance predictions. Long wavelength data obtained with quartz windows are in fairly good agreement with the predictions of Breene for the corresponding wavelength regime. Also, there appears to be a contribution to intensity in the wavelength region between the quartz and LiF cut-off values. However, this conclusion is questionable at this time because of the difficulties encountered in the use of LiF windows. A preliminary assessment of the coupling effect for the range of conditions studied suggests that it is hidden within the scatter of the present data.

The effects of the boundary layer have not been included in the present study except for the estimation of its importance in the absorption of energy 
radiated from the shock layer for a specific test condition. The boundary layer is of concern in flight situations not only because of this effect, but also because absorbed radiation will influence the boundary layer properties and, therefore, modify the convective heat transfer. This could be of particular importance for an ablating body where relatively complex molecules may exist near the surface of the body. A semi-empirical treatment of this problem, applied to the determination of the performance of ablation materials subjected to combined radiative and convective heating in superorbital flight, is given in Ref. 26. 


\section{REFERENCES}

1. Page, W.A. and Arnold, J.O.; Shock Layer Radiation of Blunt Bodies at Re-Entry Velocities; NASA TR-R-193, Apri1 1964.

2. Allen, R.A., Rose, P.H. and Camm, J.C.; Nonequilibrium and Equilibrium Radiation at Super-Satellite Re-Entry Velocities;IAS 63-77.

3. Gruszczynski, J.S., Warren, W.R. and Rogers, D. A.; Shock Tube Techniques for Studies of High Temperature Gas Radiance; Pres. 5th Shock Tube Symposium, U.S. Naval Ord. Lab., Silver Spring, Md., April 1965; also GE TIS R65SD33, August 1965.

4. Nardone, M. C, Breene, R. G., Zeldin, S.S. and Riethof, T.R.; Radiance of Species in High Temperature Air; GE TIS R63SD3, June 1963.

5. Gruszczynski, J.S. and Warren, W.R.; Experimental Heat Transfer Studies of Hypervelocity Flight in Planetary Atmospheres; AIAA Paper No. 63-450, Aug. 1963. Also AIAA Journal, Vol. 2, No. 9, pp. 15421550, Sept. 1964.

6. Sherman, M.P. and Kulander, J.L.; Free-Bound Radiation from Nitrogen, Oxygen, and Air; GE TIS R65SD15, May 1965.

7. Hahne, G. E. ; The Vacuum Ultraviolet Radiation from $\mathrm{N}^{+}$and $\mathrm{O}^{+}$Electron Recombination in High-Temperature Air; NASA TN D-2794, June 1965.

8. Biberman, L.M., Vorobev, V.S., Norman, G. E. and Iakubov, I. T.; Radiation Heating in Hypersonic Flow; Kosmich Issled, Vol. 2, No. 3, pp. $441-454,1964$. 
9. Serbin, H.; Supersonic Flow Around Blunt Bodies; Journal of the Aeronautical Sciences, Vol. 25, pp. 58-59, January 1958.

10. Goulard, R.; The Coupling of Radiation and Convection in Detached Shock Layers; J. Quant. Spectrosc. Radiat. Transfer, Vol. 1, pp. 249-257, February 1961 .

11. Howe, J.T. and Viegas, J.R.; Solutions of the Ionized Radiating Shock Layer, Including Reabsorption and Foreign Species Effects, and Stagnation Region Heat Transfer; NASA TR R-159, 1963.

12. Wilson, K.H. and Hoshizaki, H.; Inviscid, Nonadiabatic Flow About Blunt Bodies; AIAA Journal, Vol. 3, No. 1, January 1965.

13. Hoshizaki, H. and Wilson, K.H.; The Viscous, Radiating Shock Layer About a Blunt Body; AIAA Journal, Vol. 3, No. 9, September 1965.

14. Hoshizaki, H. and Wilson, K. H.; private communication; August 1965.

15. Sherman, M.; private communication; October 1965.

16. Warren, W.R., Rogers, D. A. and Harris, C.J.; The Development of an Electrically Heated Shock Driven Test Facility; GE TIS R62SD37, April 1962. Also Proc. 2nd Symp. on Hypervelocity Tech., Denver Res. Inst., Denver, March 1962.

17. Camm. J.; Escape Velocity Shock Tube with Arc Heated Driver; 2nd Symp. on Hypervelocity Techniques, Univ, of Denver, Denver, Colo., March 1962.

18. Gruszczynski, J.S., Warren, W.R. and Diaconis, N.S.; Laboratory Simulation of Hypervelocity Heat Transfer Problem During Planetary Entry; GE TIS R64SD73, Nov. 1964. 
19. Gruszczynski, J.S. and Rogers, D. A.; Shock Tube Instrumentation Techniques for Study of Hypervelocity Entry Problems; GE TIS R64SD67, Sept. 1964.

20. Gruszczynski, J.S., Harris, C.J., Rogers, D. A. and Warren, W. R.; Fast Response Total Radiation Gage for Measurement of Radiant Emis sion from High Temperature Gas, GE TIS R63SD11, January 1963. Also IEEE Conf. Paper No. 63-438, January 1963.

21. Vorobev, V.S. and Norman, G. E.; Energy Radiated in Spectral Lines by an Equilibrium Plasma; Optical Spectr. $17,180,1964$.

22. Nerem, R.M.; Radiating Flows Around Re-Entry Bodies; Presented at the XVIth International Astronautical Congress, Athens, Greece, September 13-18, 1965; Reprint No. 1941-1, Sept. 1965.

23. Hoshizaki, H.; Equilibrium Total Radiation Measurements in Air at Superorbital Entry Velocities; Lockheed Missiles and Space Co., Rept. No. 6-90-63-97, October 1963 .

24. Nerem, R.M. and Stickford, G.H.; Shock Tube Studies of Equilibrium Air Radiation; AIAA Journal, Vol. 3, No. 6, June 1965.

25. Cooper, D. M.; High-Resolution Experimental Spectra and Theoretical Studies of Equilibrium Radiation from Blunt Body Travelling at 37,000 ft/sec.; AIAA Paper No. 66-104, Presented at the 3rd Aerospace Sciences Meeting, New York, 1966.

26. Diaconis, N.S., Warren, W.R. and Shaw, T.E.; The Hypervelocity Heat Protection Problem; Presented at XVI Inter. Ast. Congress, Athens, Sept. 1965. Also GE TIS R65SD60, Nov. 1965. 


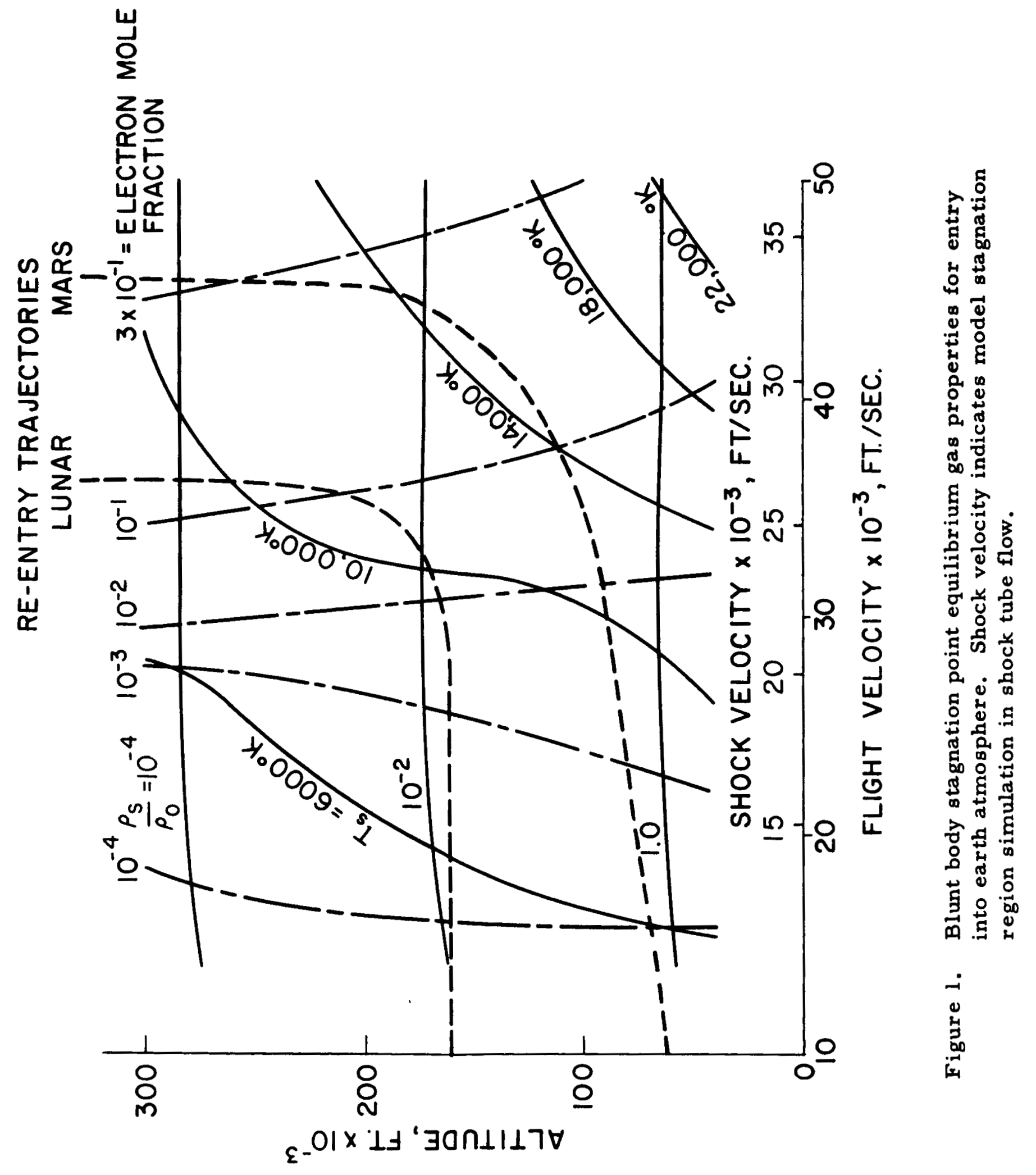




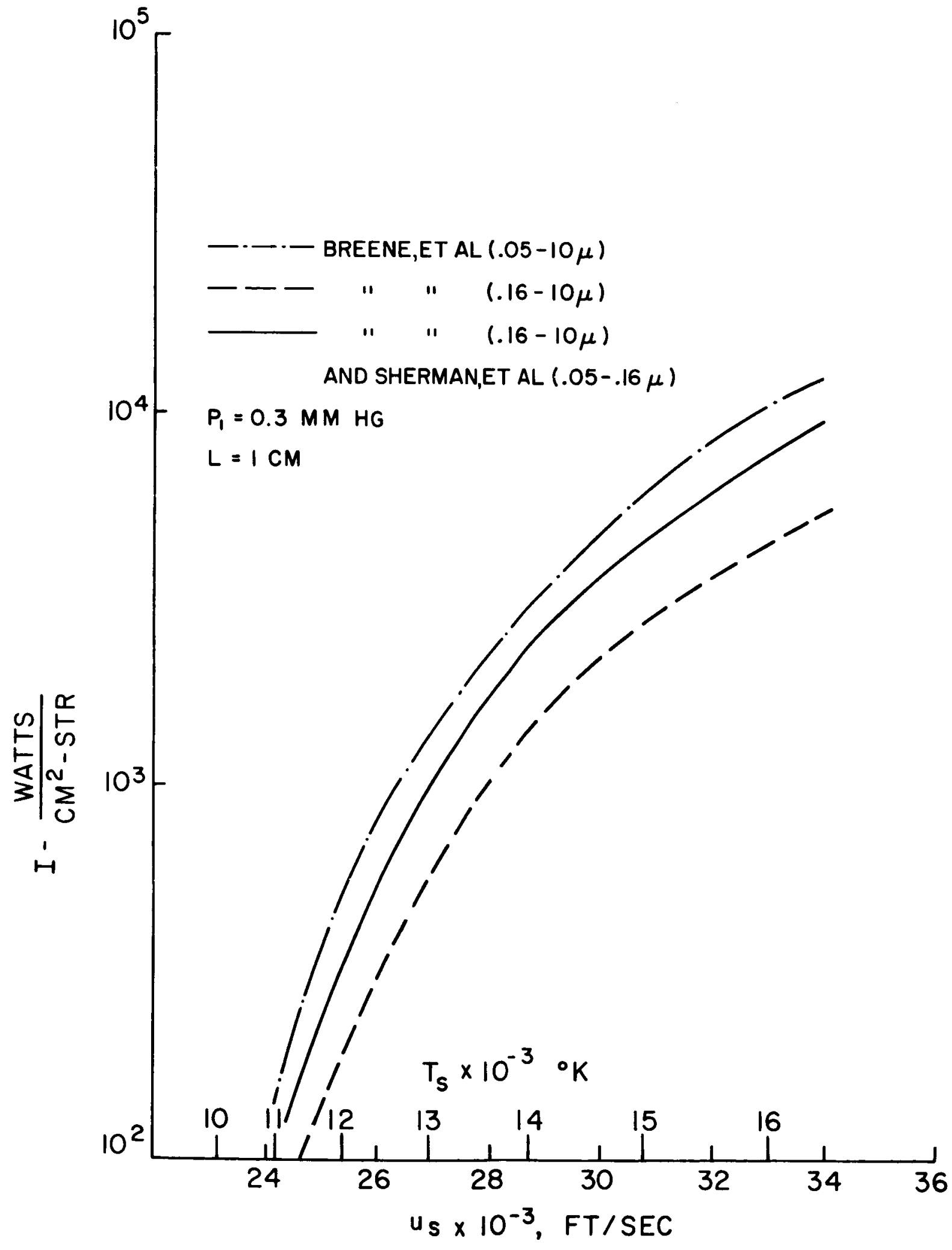

Figure 2. Radiative intensity of air for blunt model stagnation conditions in a shock tube. 


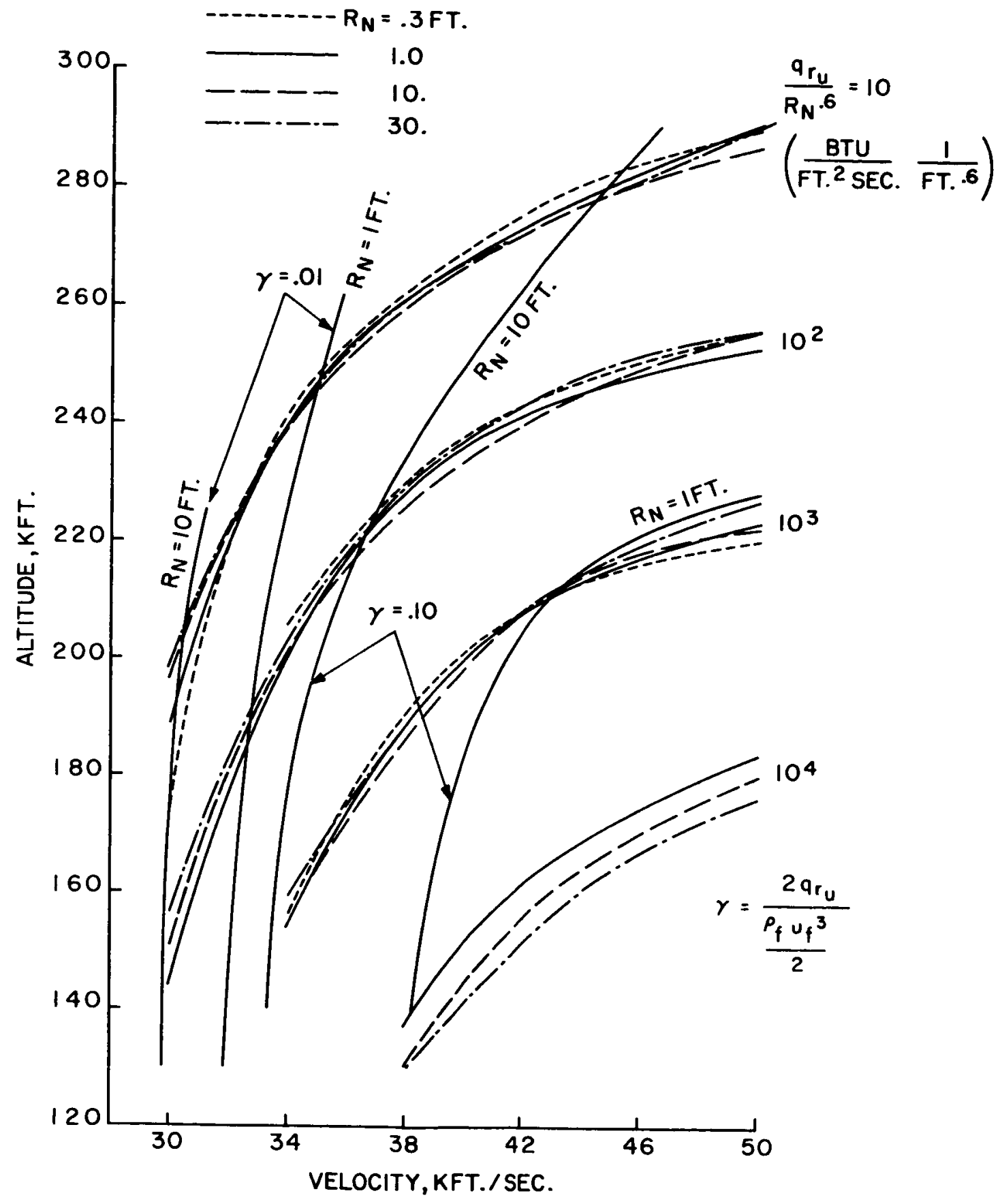

Figure 3. Correlation of the Biberman, et al predictions of unocoupled stagnation point radiative heating for a range of body nose radii. 


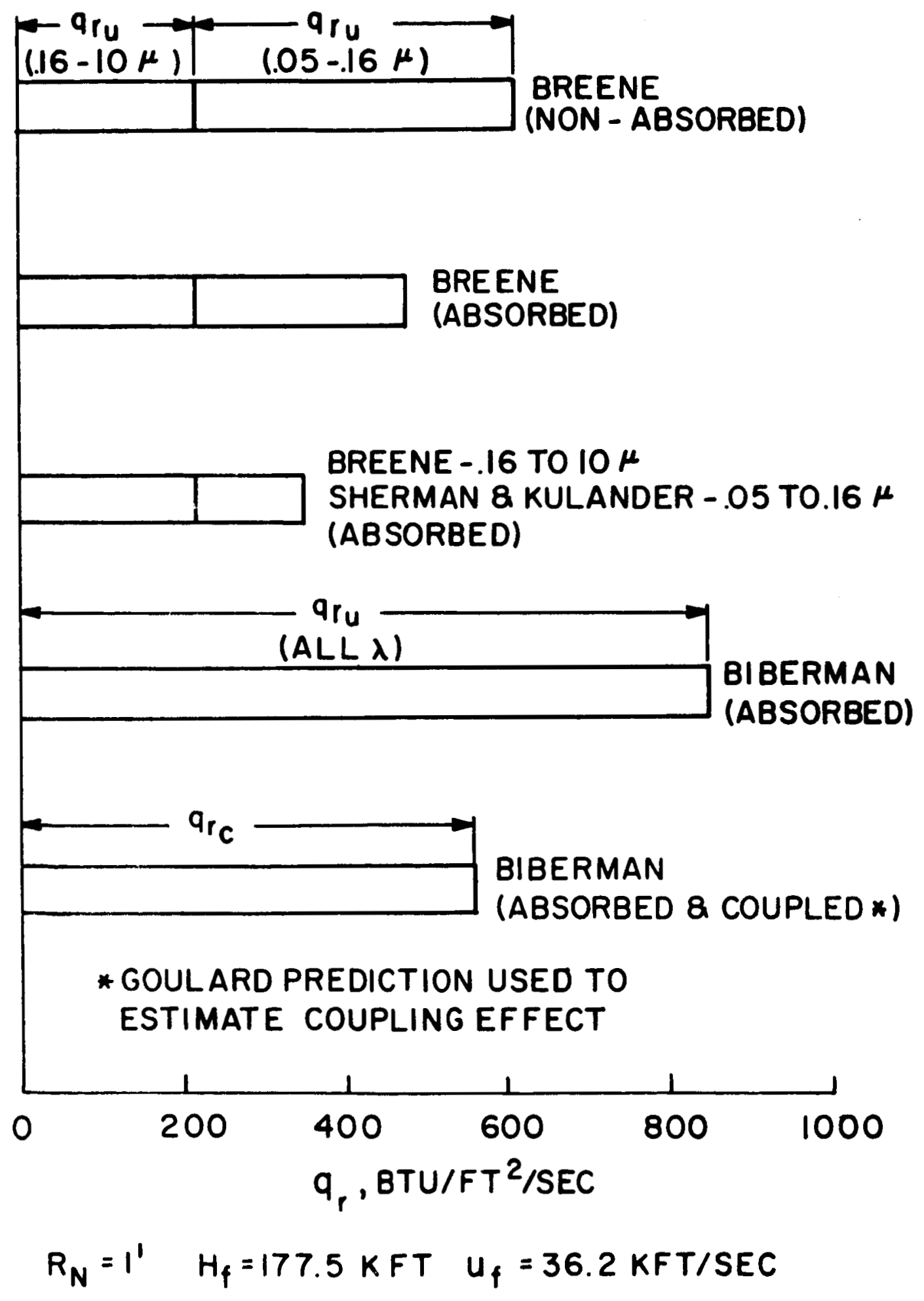

Figure 4. Comparison of the radiative heating predictions of several investigators indicating the effects of self-absorption and coupling. 


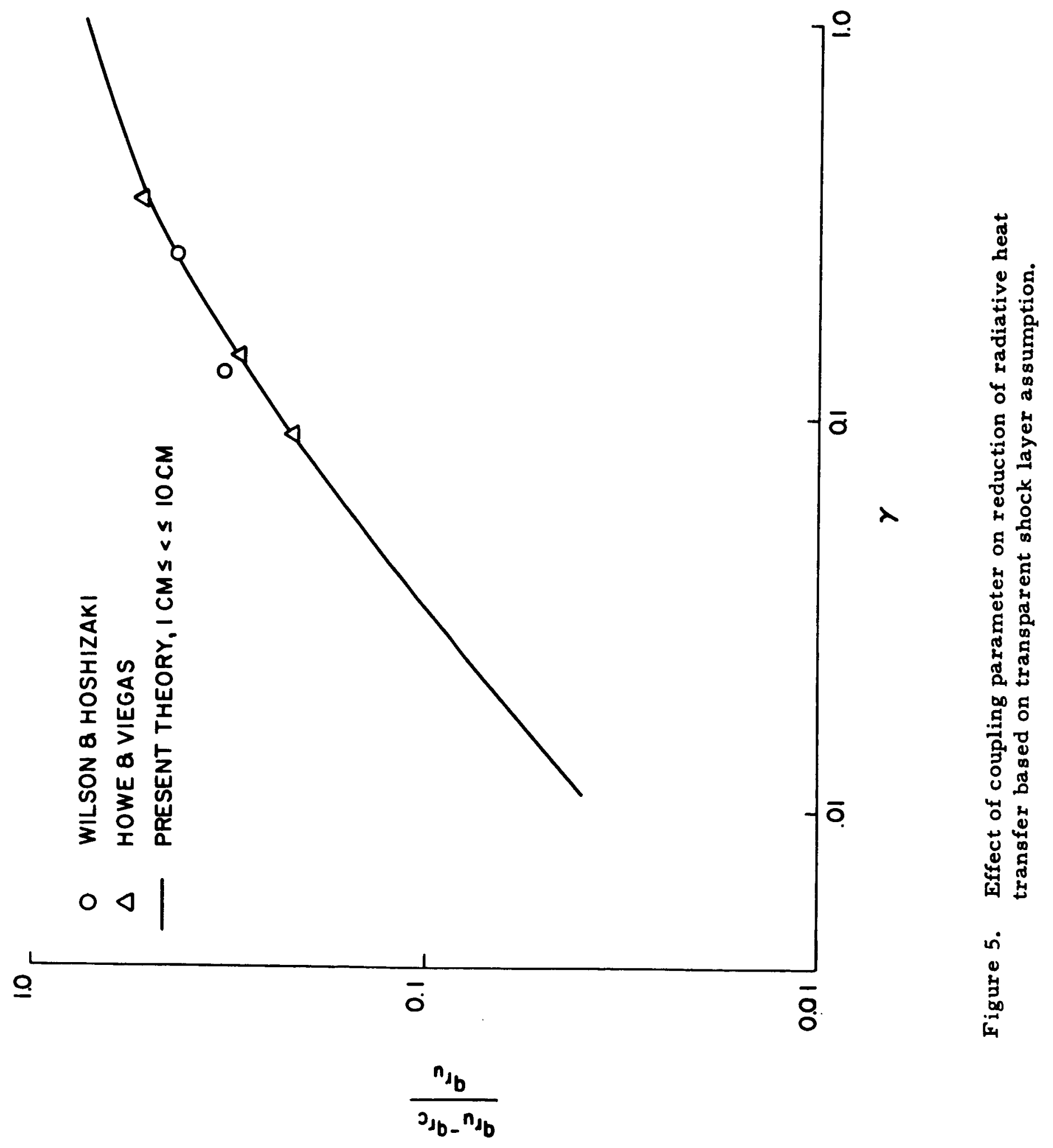




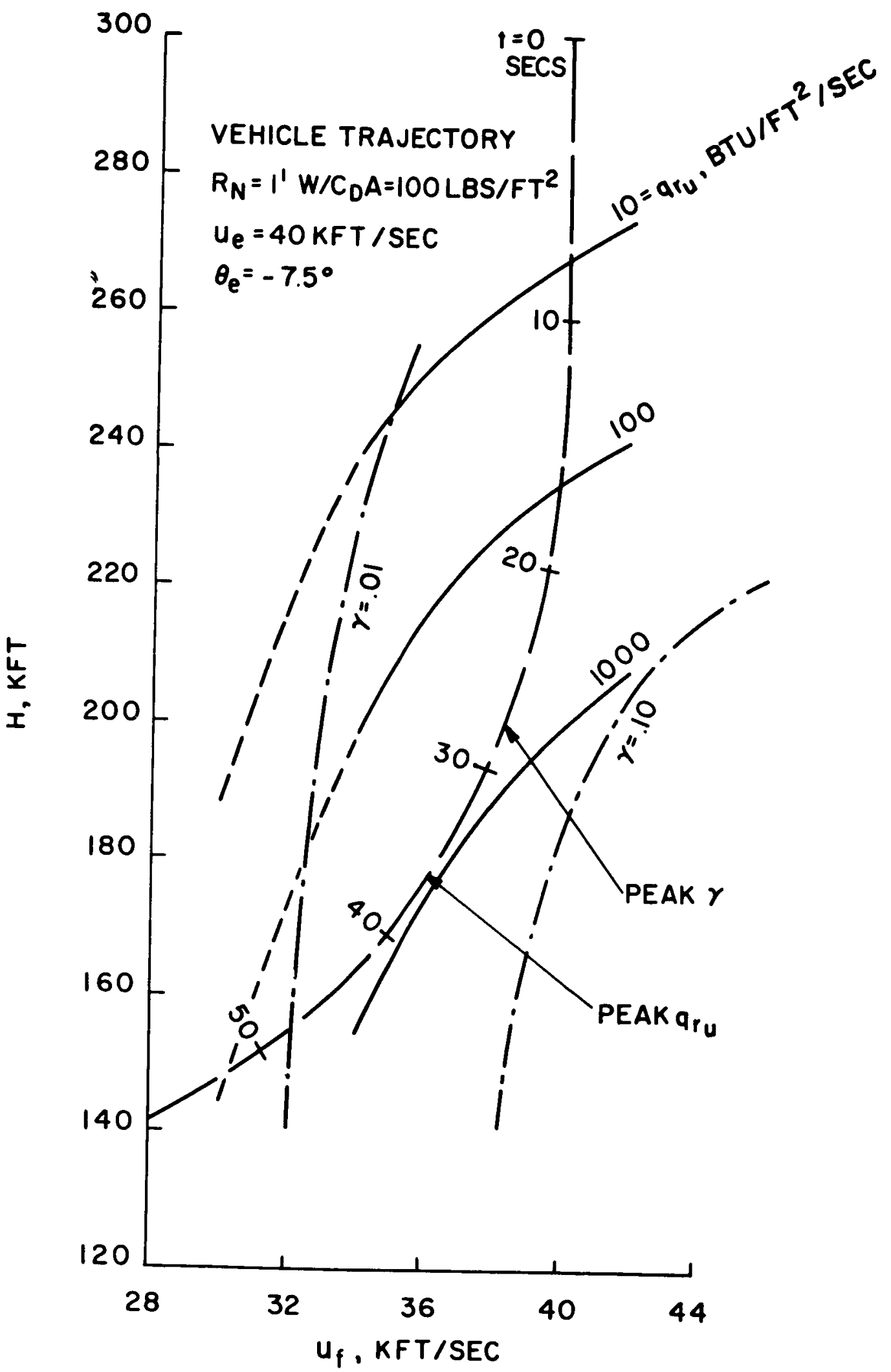

Figure 6. Re-entry vehicle trajectory showing positions of peak uncoupled stagnation point radiative heating and peak coupling parameter (Biberman, et al predictions). 


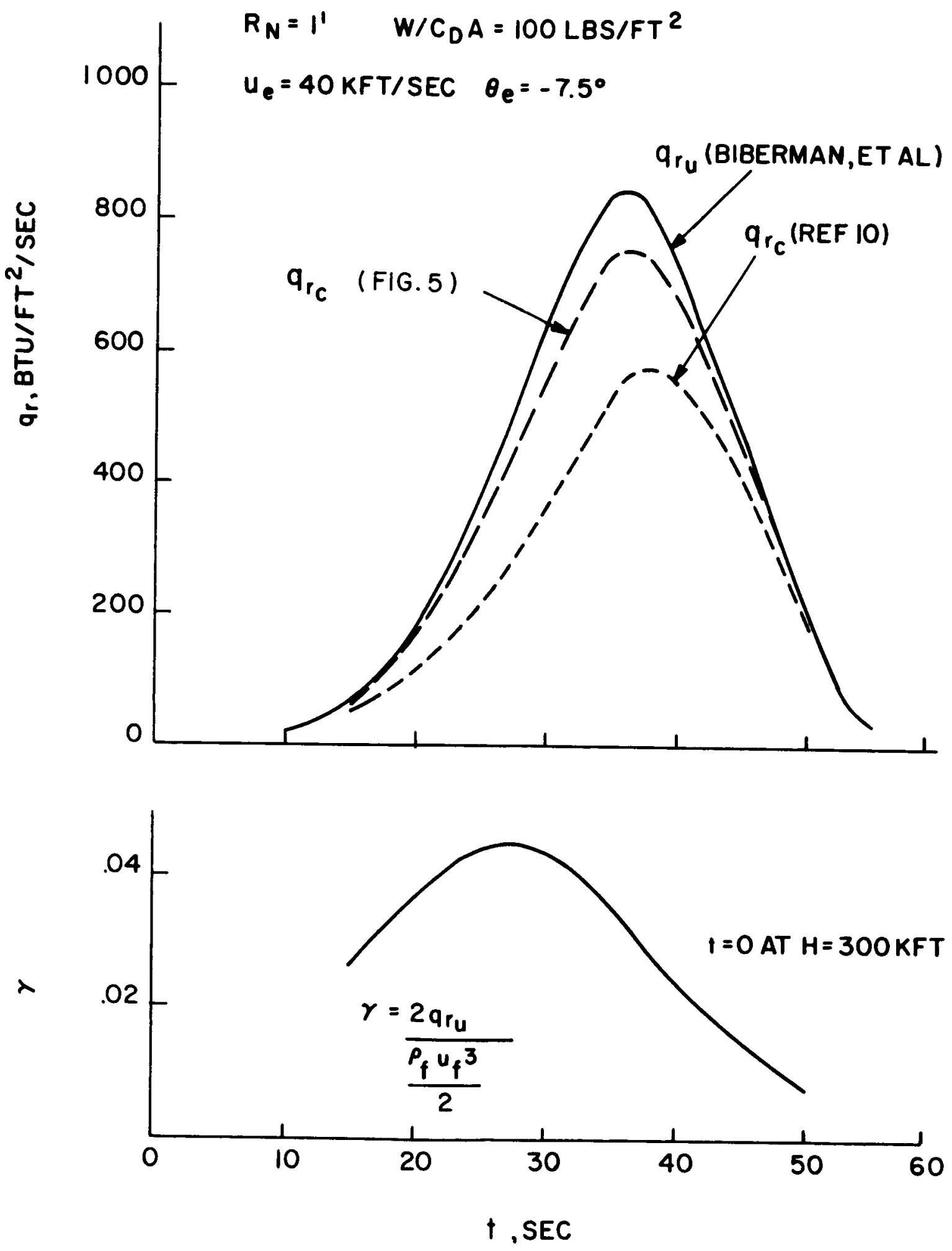

Figure 7. Variation of uncoupled radiative heat transfer, $q_{r_{u}}$, and coupling parameter, for vehicle trajectory of Fig. 6. Reductions in radiative heating, as predicted by three approximate analyses, are shown. 


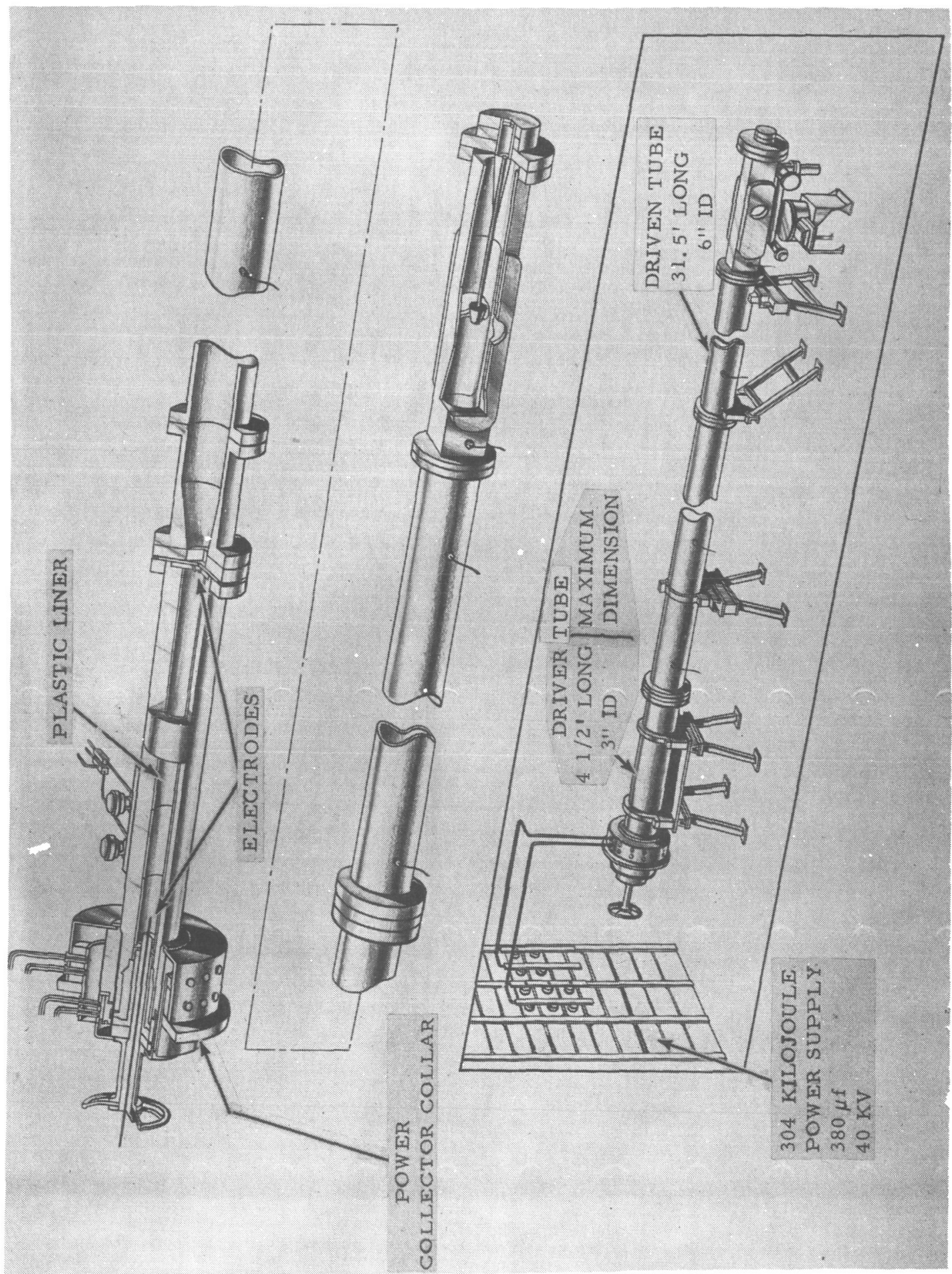



$\underbrace{\infty}_{\substack{0 \\ 0 \\ 0.1}}$ 


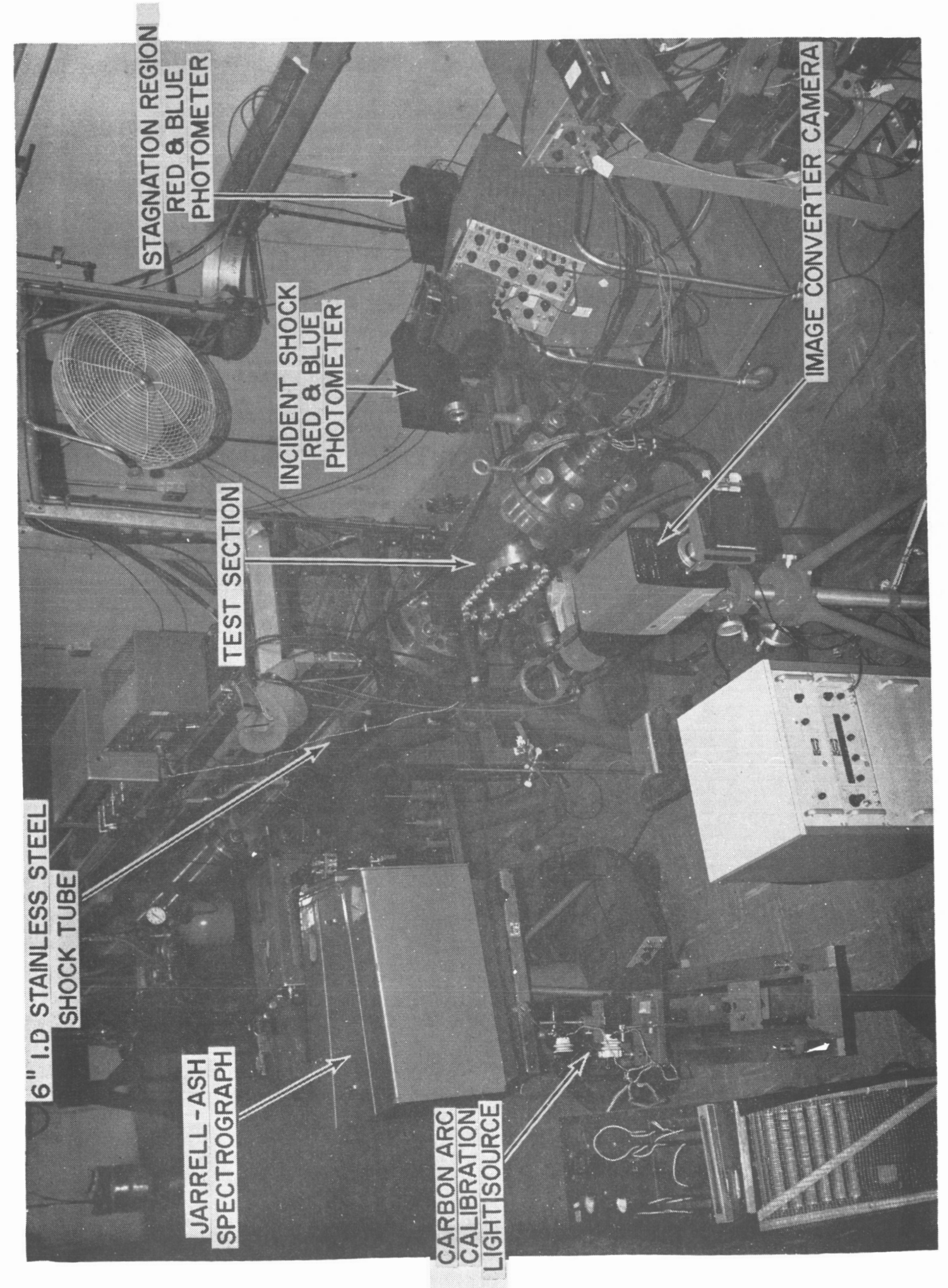

 


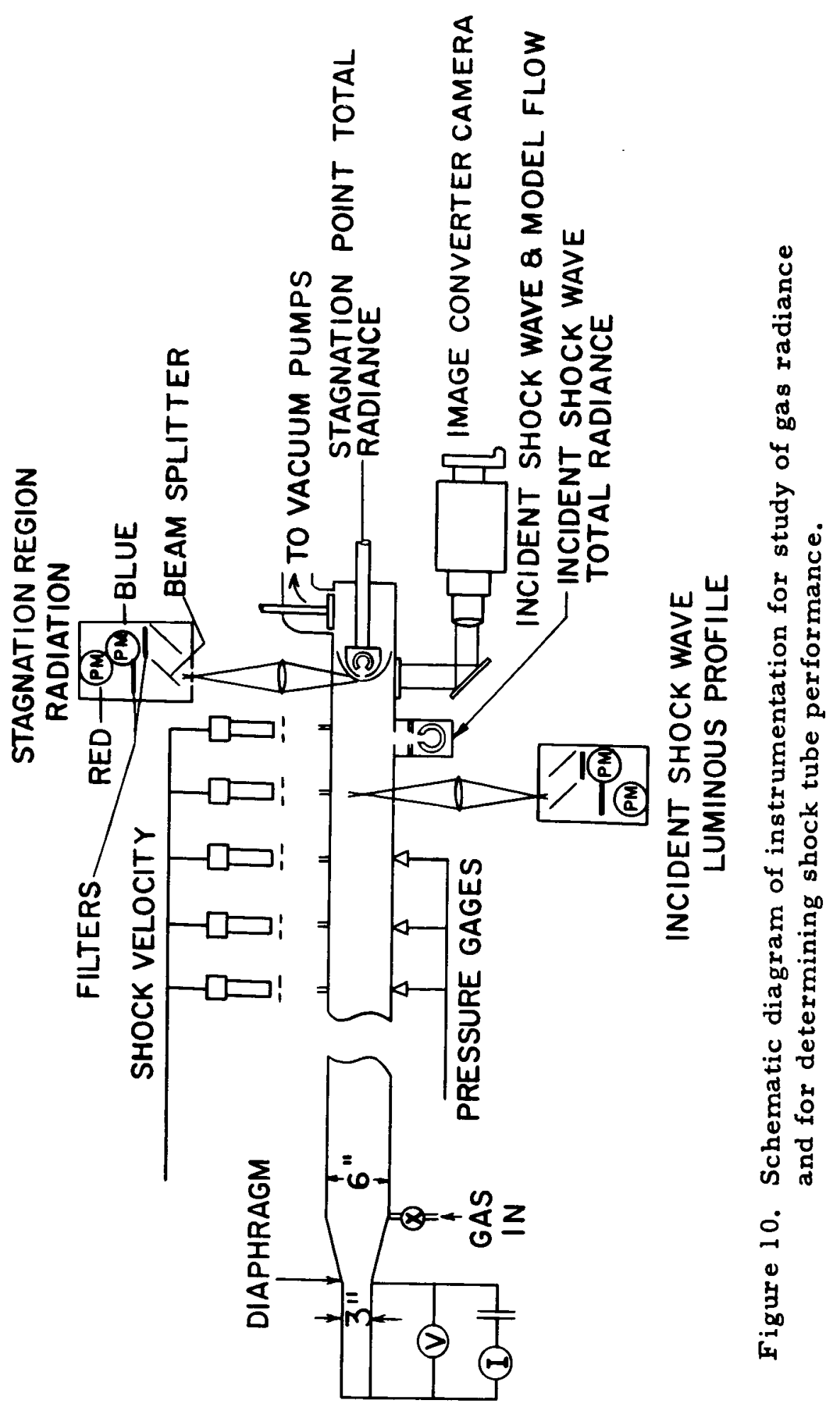




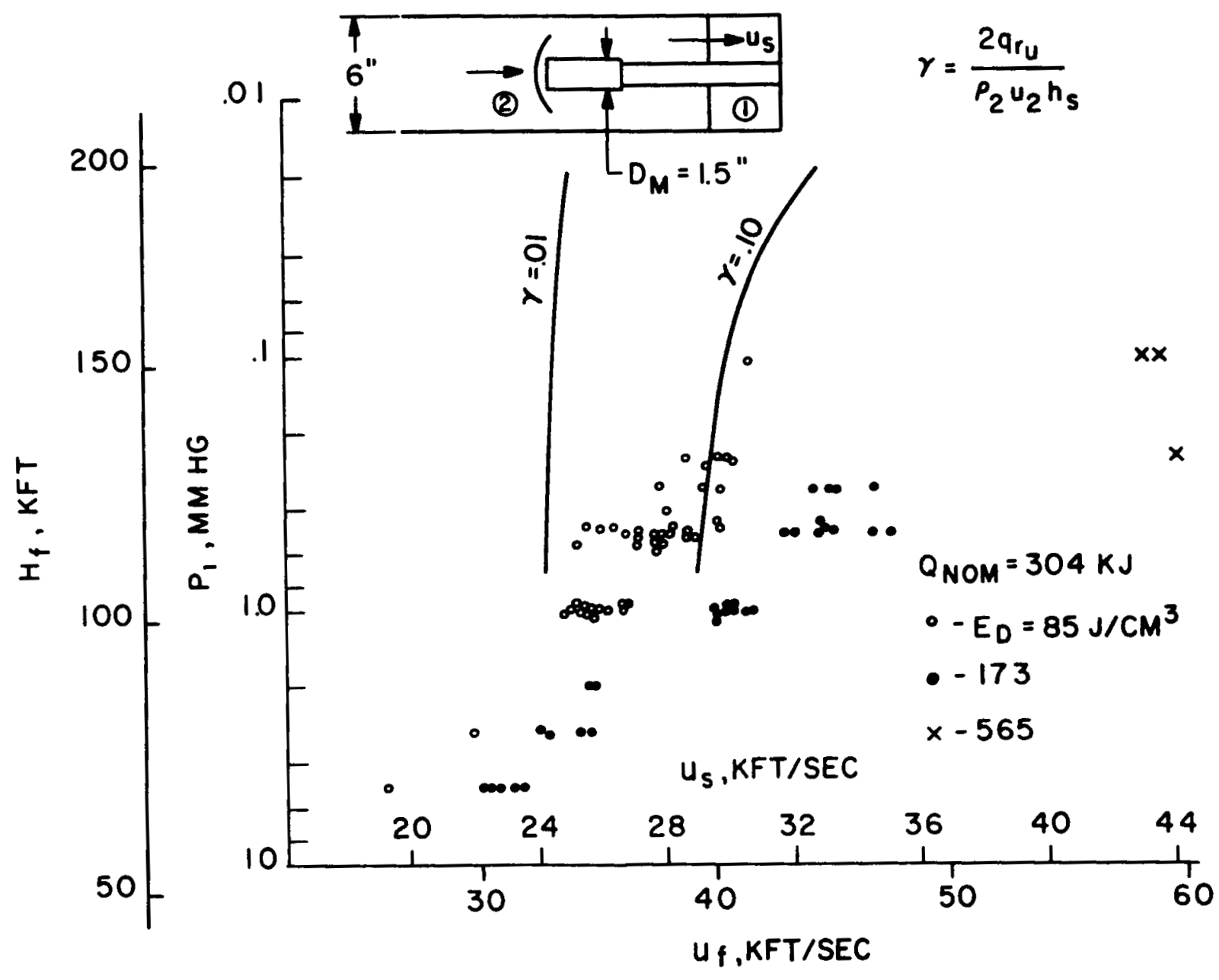

Figure 11. Shock velocity - initial pressure performance for electrically driven shock tube showing representative data for three different driver geometries, specified by nominal driver gas energy density, $E_{D}$. The coupling parameter, $\gamma$, based on the Biberman, et al predictions, is given for one model geometry. The simulated flight velocity and altitude scales are for the blunt model shock tube test configuration. 


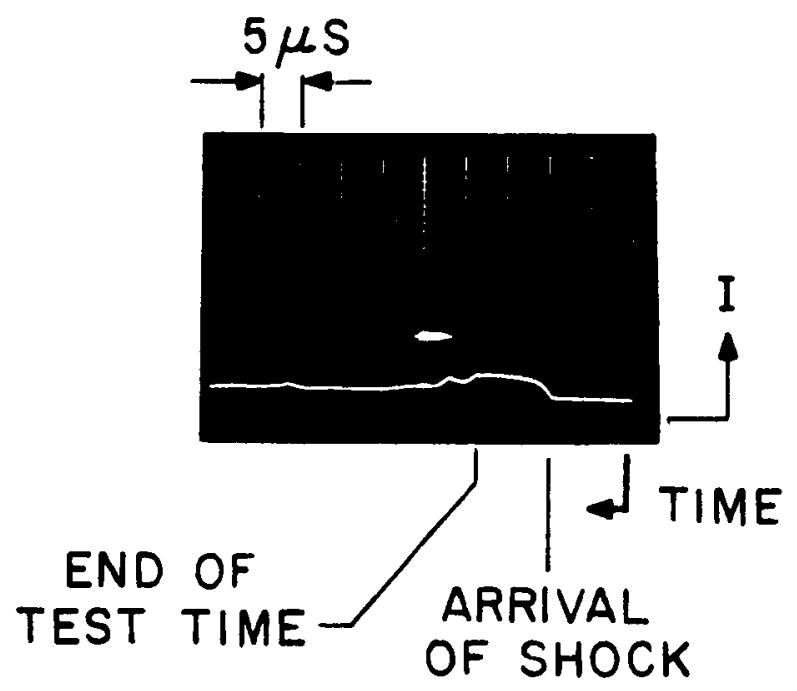

$$
\begin{aligned}
& P_{1}=0.25 \mathrm{MM} \mathrm{Hg} \\
& U_{S}=44,000 \mathrm{FT} / \mathrm{SEC}
\end{aligned}
$$

Figure 12. Oscilloscope trace of signal from photomultiplier vieving model stagnation flow from sidewall of shock tube. 

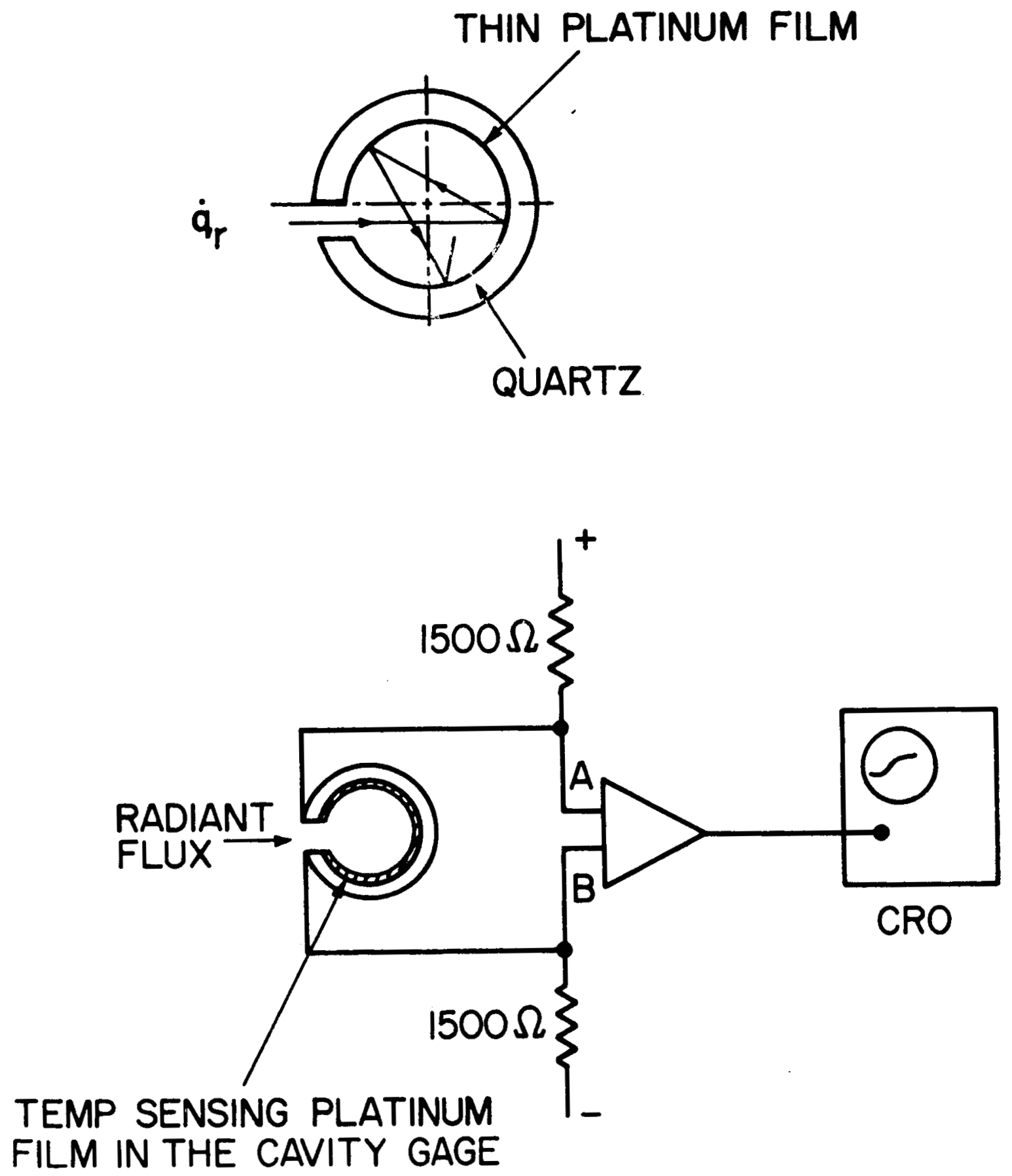

Figure 13. Total radiation cavity gage. The geometry of cylindrical section and schematic of gage electric circuit are shown. 


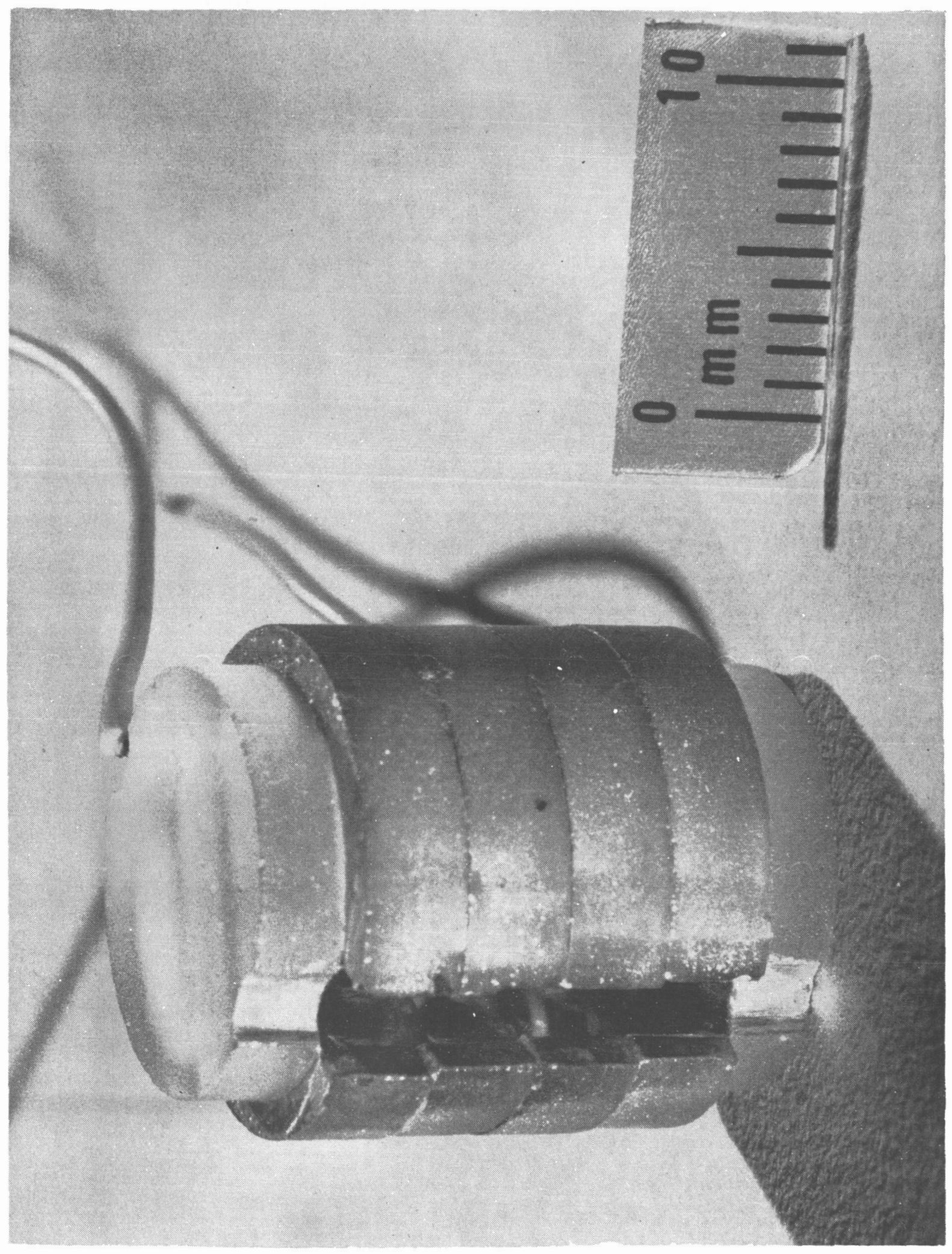

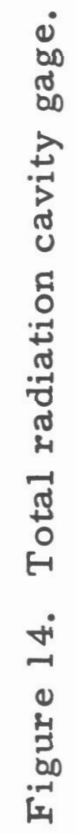



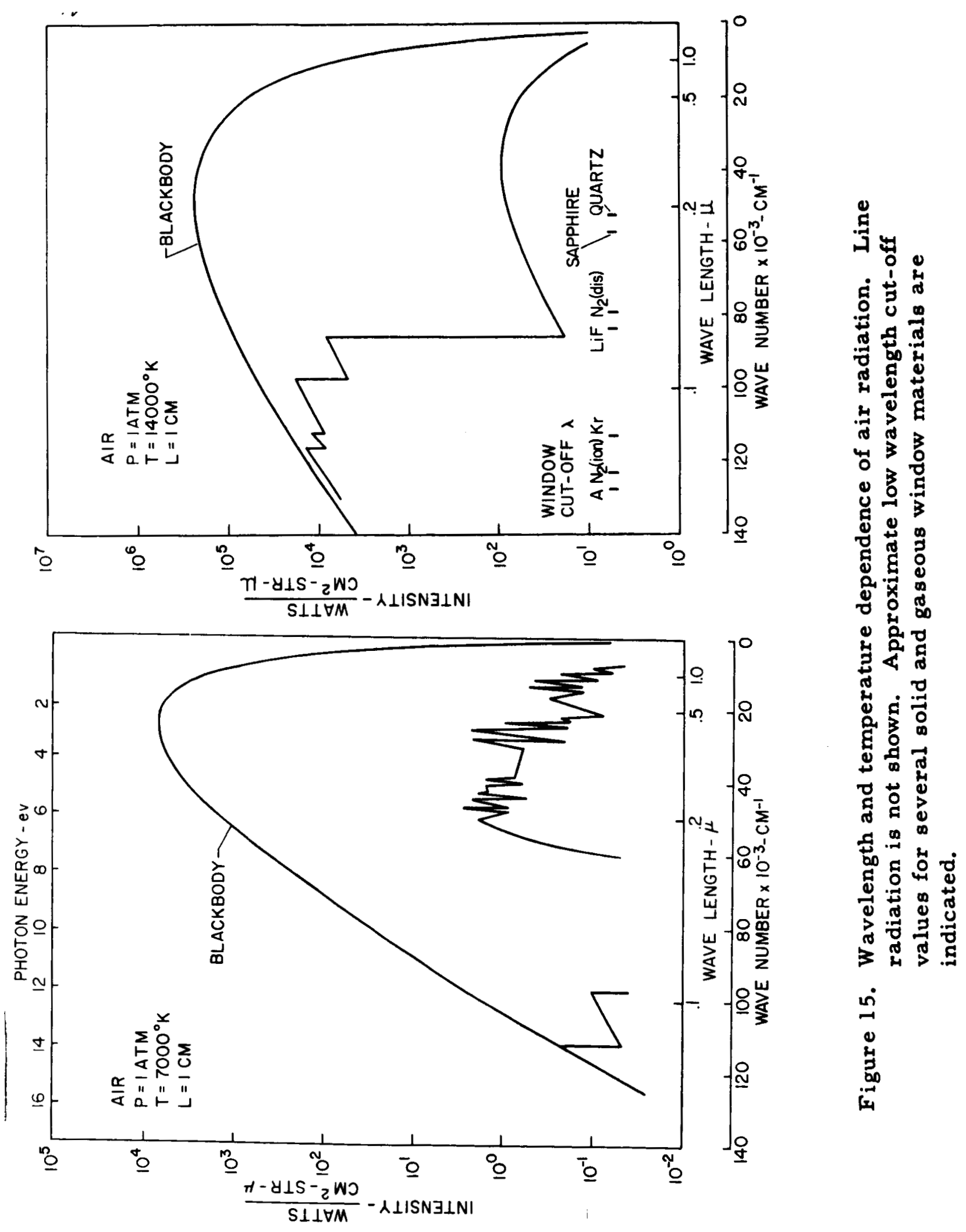

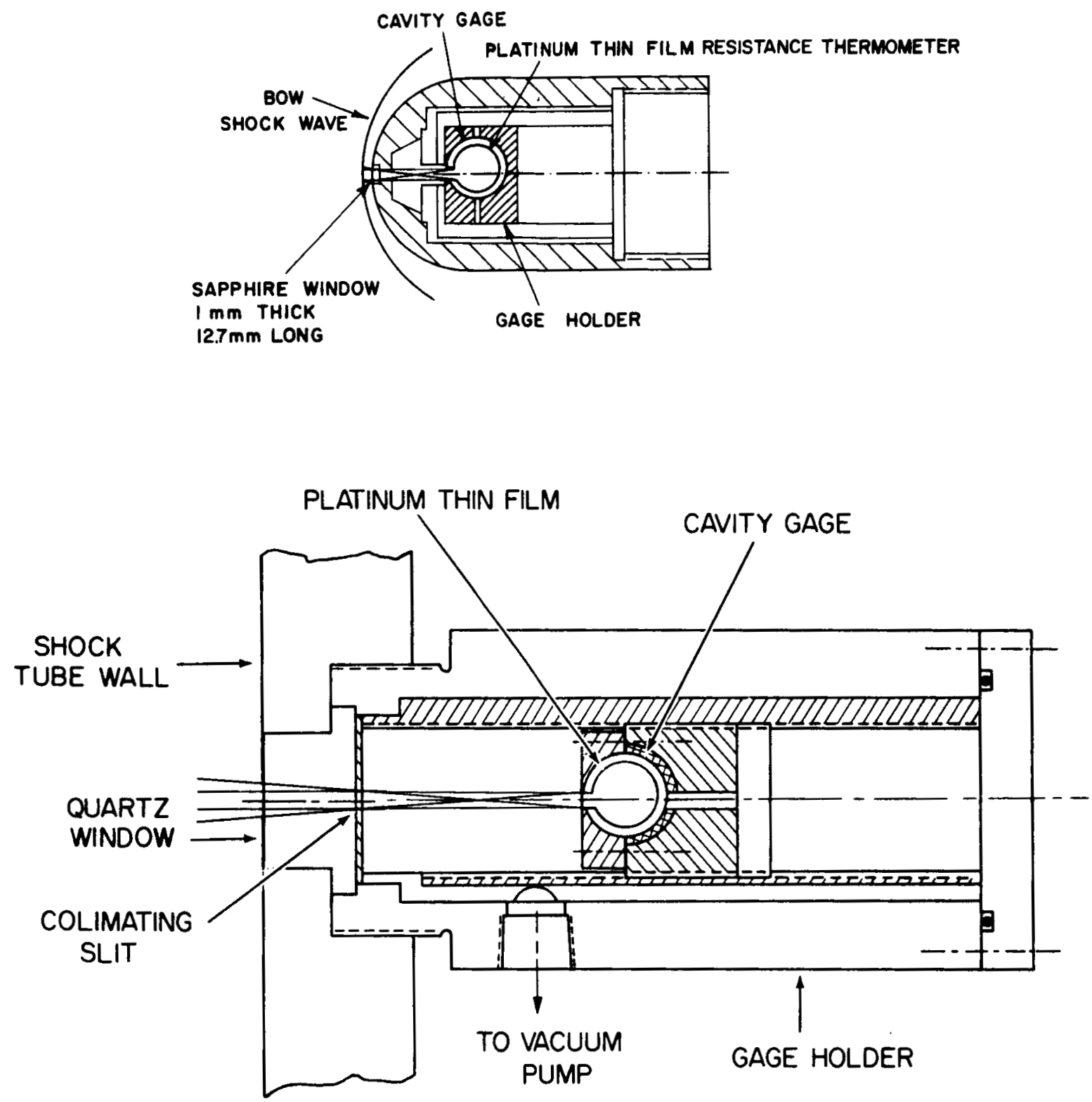

SIDEWALL CONFIGURATION

Figure 16. Total radiation cavity gage-model system with wolid windows used for radiation measurements. The upper drawing shows stagnation flow model configuration. The lower drawing indicates sidewall a rrangement. 


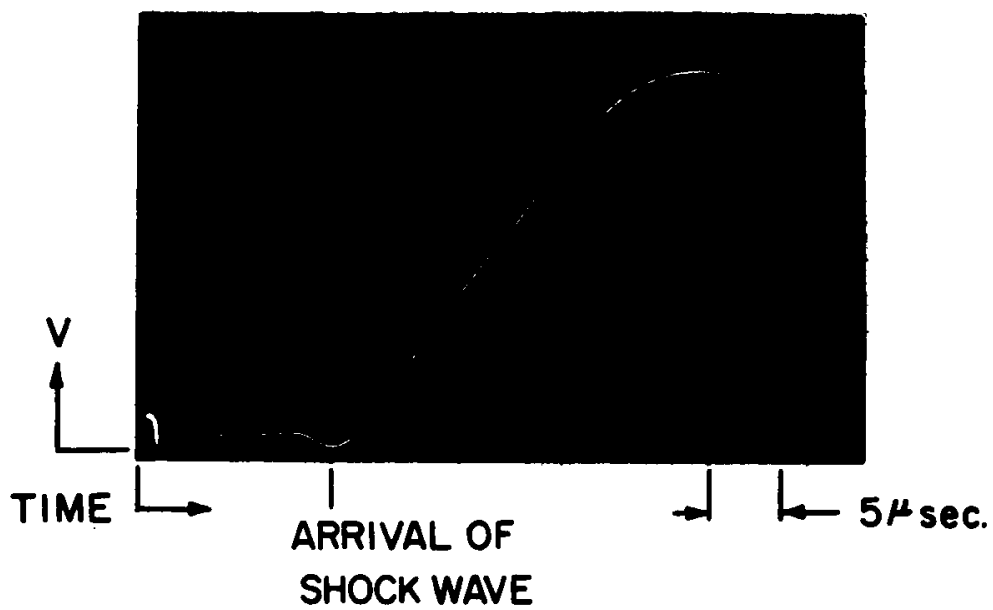

$$
P_{1}=0.33 \mathrm{~mm} \mathrm{Hg} \quad U_{S}=29,500 \mathrm{ft} / \mathrm{sec}
$$

CAVITY GAGE WITH LIF WINDOW

MODEL GAS $\mathrm{N}_{2}$ AT I ATM PRESSURE

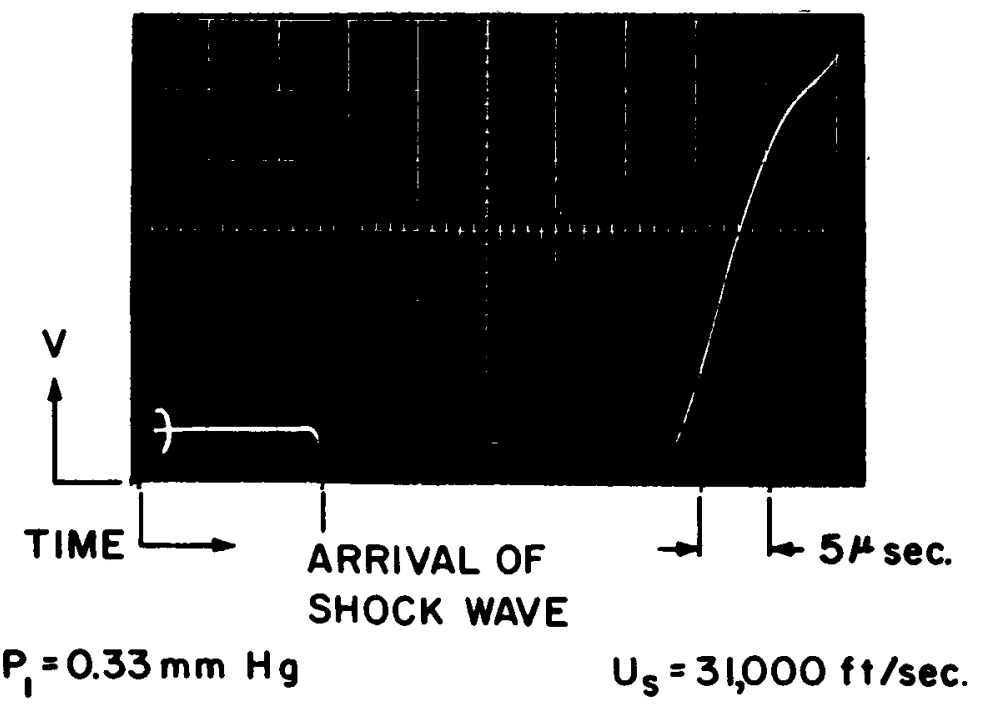

CAVITY GAGE WITH LIF WINDOW MODEL GAS - A AT 2ATM PRESSURE

Figure 17. Oscilloscope traces of cavity gage response with LiF window. Upper photograph shows model filled with pure $\mathrm{N}_{2}$. Lower photograph shows strong photoelectric effect when model was filled with argon. 


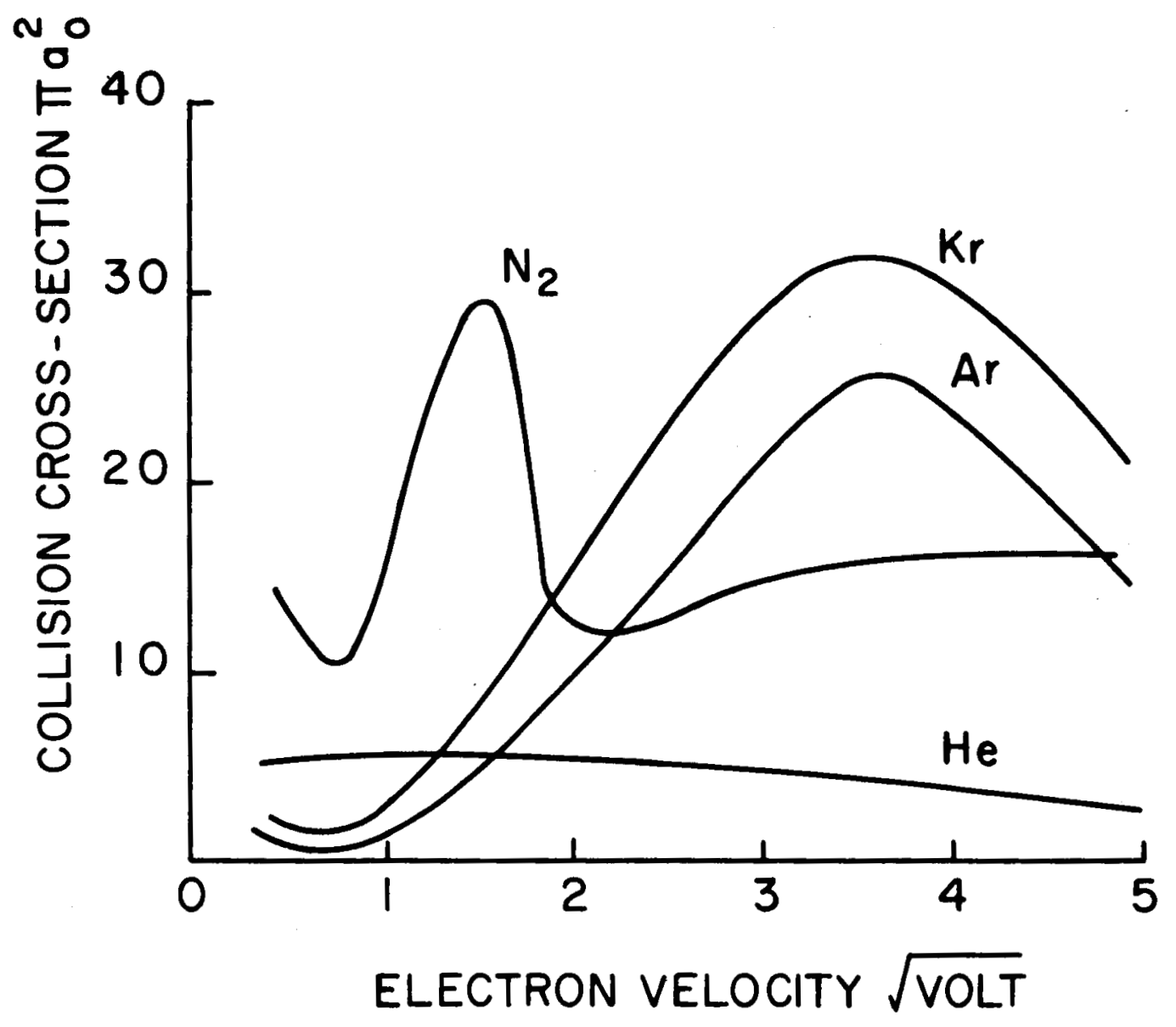

Figure 18. Total collision cross-sections of several gases for electrons with various energies. 


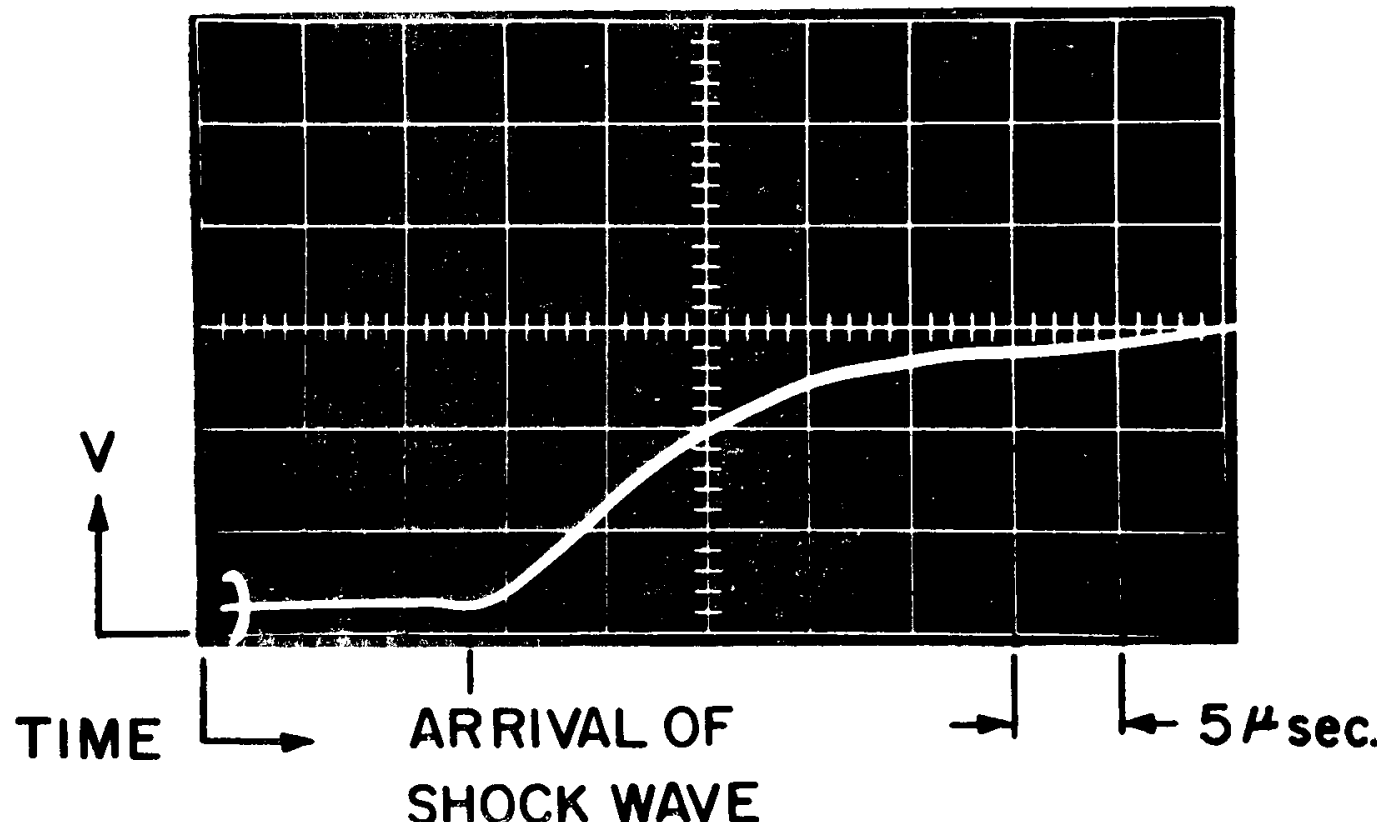

$P_{1}=0.33 \mathrm{~mm} \mathrm{Hg}$

$U_{s}=30,500 \mathrm{ft} / \mathrm{sec}$

\section{CAVITY GAGE WITH LIF WINDOW MODEL GAS $50 \% \mathrm{He}-50 \% \mathrm{Kr}$ PRESSURE 3 ATM}

Figure 19. Oscilloscope trace of cavity gage response with LiF window. Model filled with $50 \% \mathrm{Kr}-50 \% \mathrm{He}$ gas mixture. 

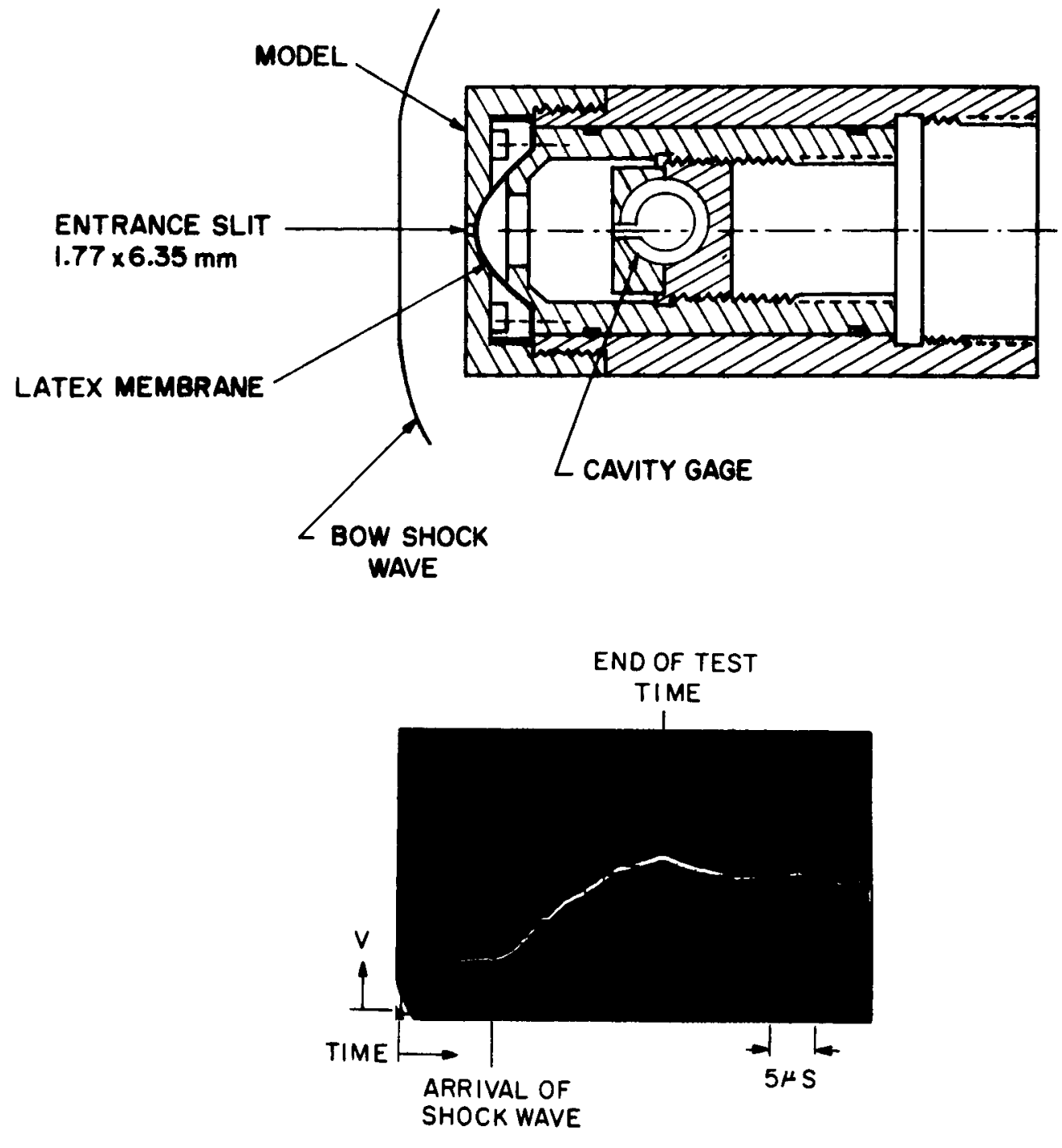

$P_{1}=0.33 \mathrm{MM} \mathrm{HG} \quad U_{S}=27,850 \mathrm{FT} / \mathrm{SEC}$

MODEL GAS : He - Kr

Figure 20. Windowless cavity gage - model system and gage responst shock tube test. 

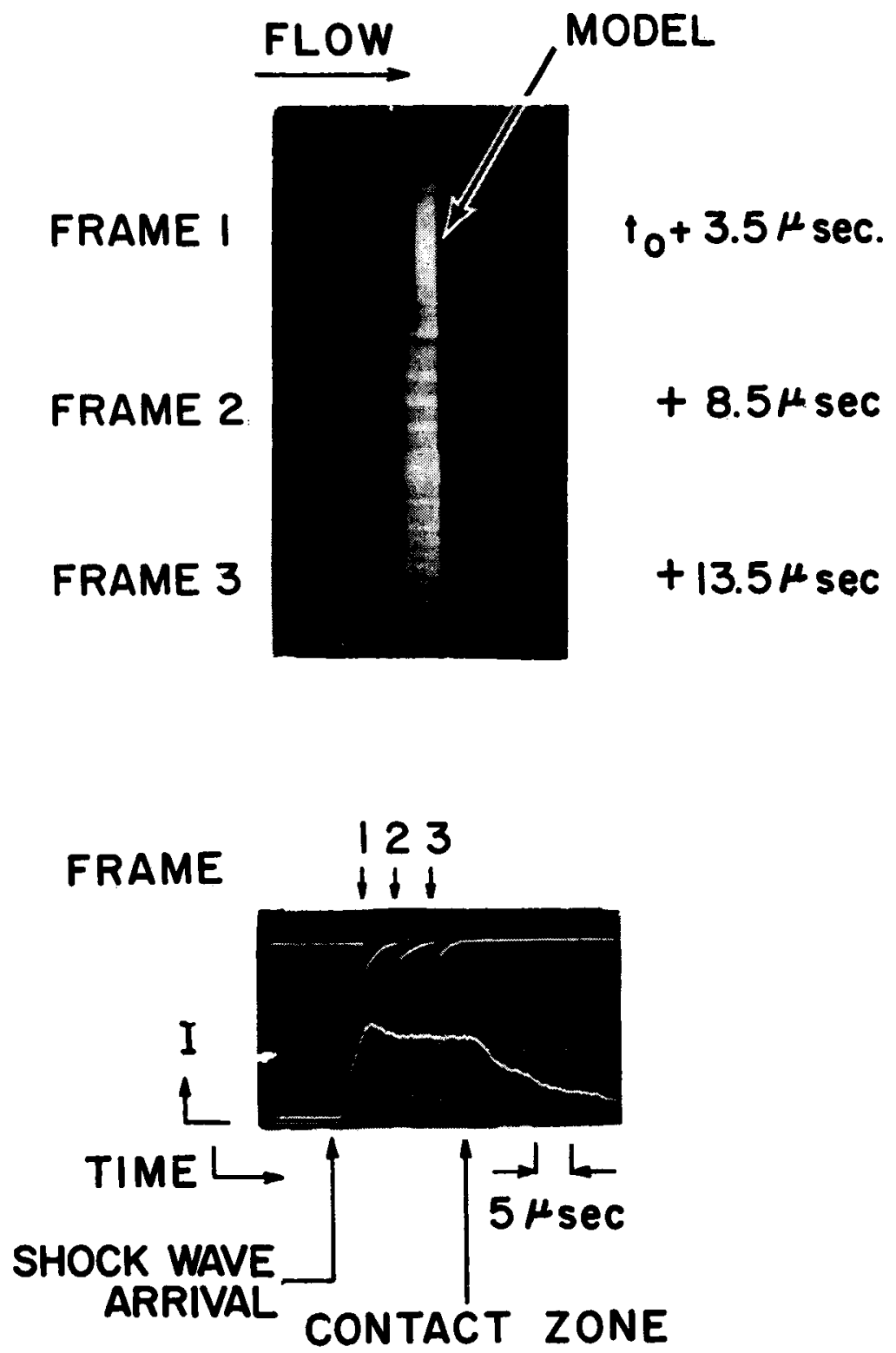

Figure 21. Image converter camera photographs of stagnation region shock layer taken from sidewall. Lower photograph shows signal from camera monitor and response of photomultiplier viewing stagnation region flow from sidewall. The incident shock velocity and initial tube pressure were $28,500 \mathrm{ft} / \mathrm{sec}$ and $.33 \mathrm{~mm} \mathrm{hg}$, respectively. 


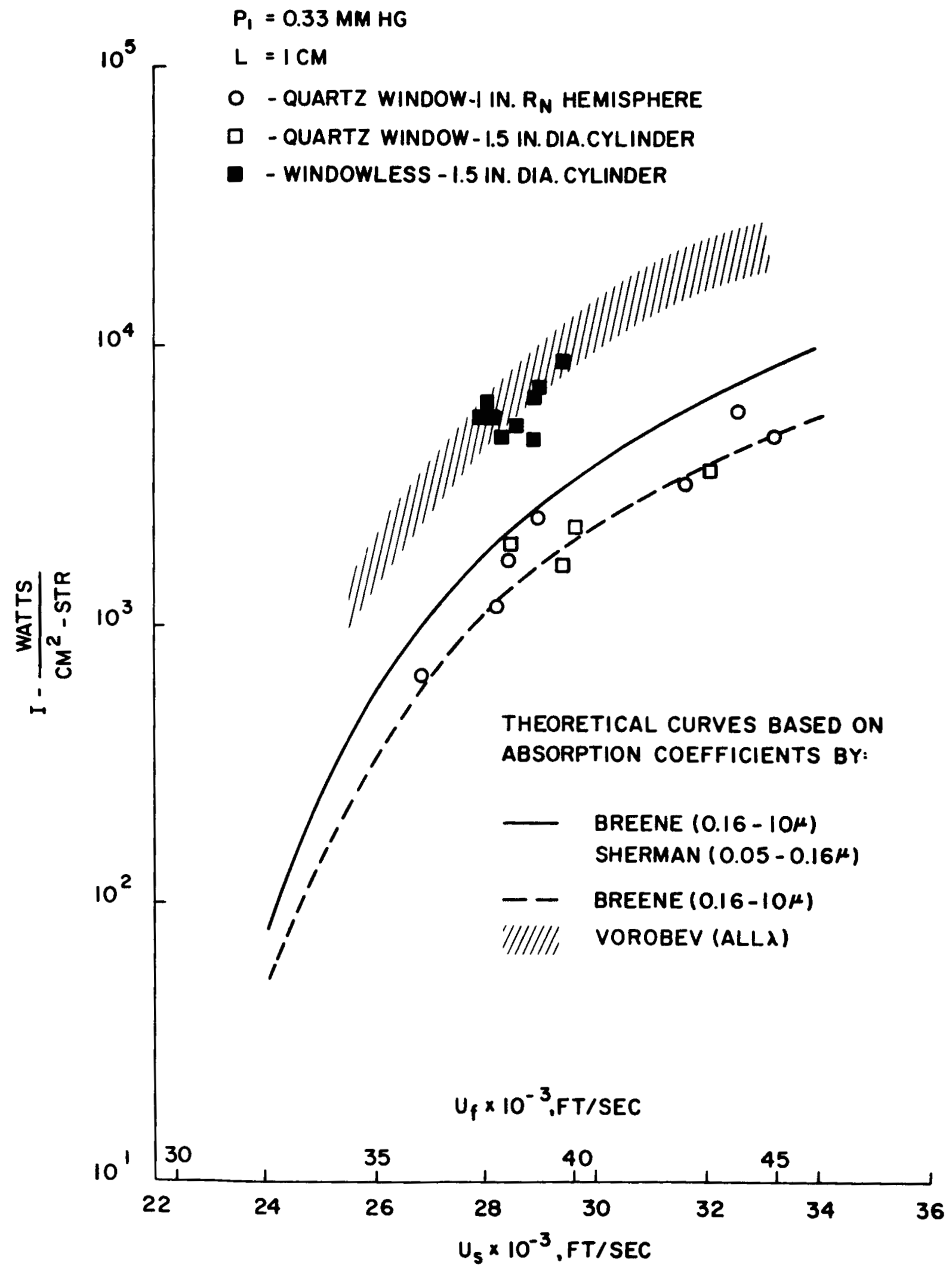

Figure 22. Comparison of experiment and theory for window and windowless gage-model configurations. 


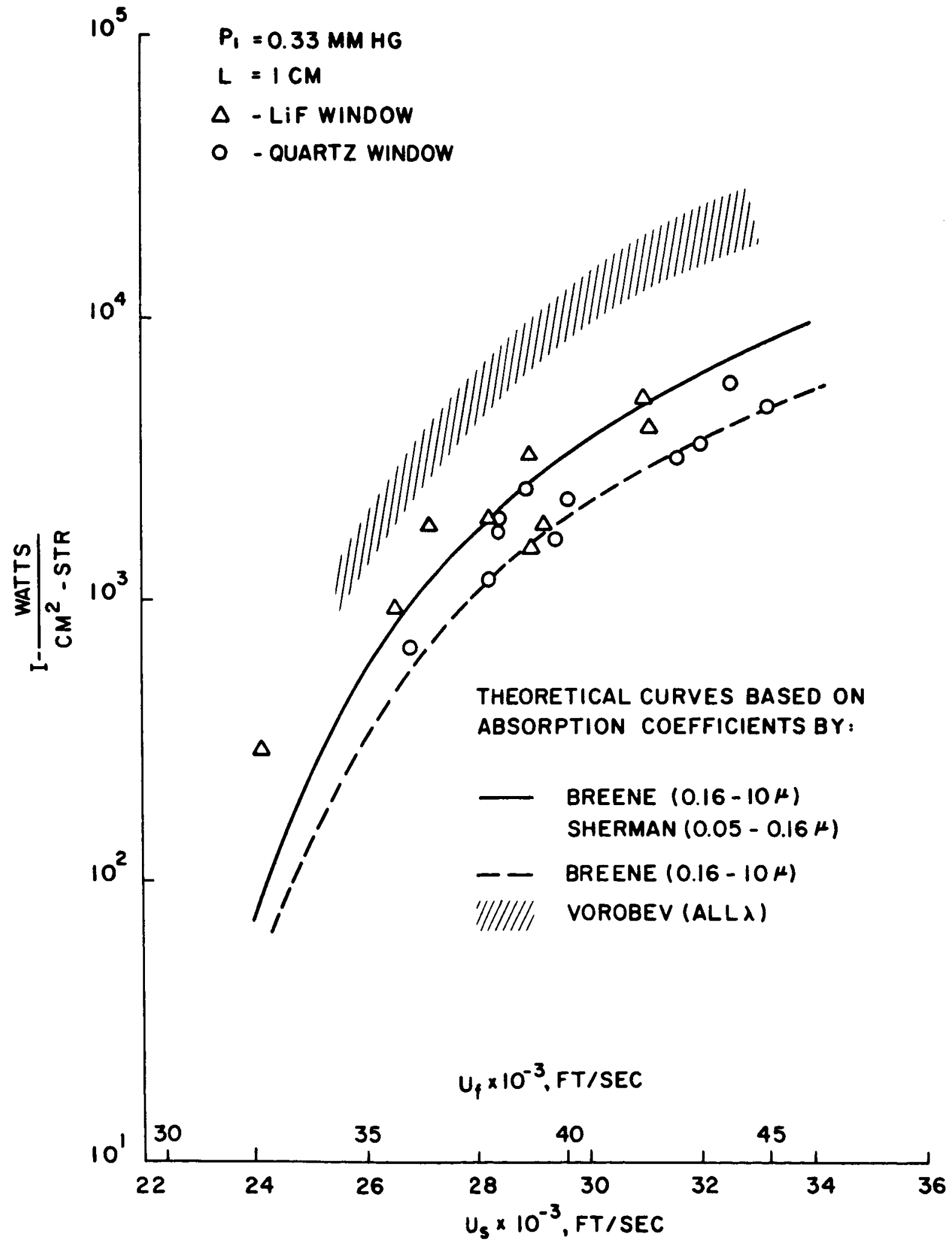

Figure 23. Comprarison of experimental data for quartz and lithium fluoride windows. 